LAWRENCE LIVERMORE N A TION AL LABORATORY

\title{
A Review of the U.S. Department of Energy's Inertial Fusion Energy Program
}

R. K. Linford

April 29, 2004

Journal of Fusion Energy 
UCRL-JRNL-203886

\title{
A Review of the U. S. Department of Energy's Inertial Fusion Energy Program
}

\author{
Rulon Linford, University of California (retired), Chair \\ Riccardo Betti, University of Rochester, Vice Chair \\ Jill Dahlburg, Naval Research Laboratory, Vice Chair \\ James Asay, Washington State University \\ Michael Campbell, General Atomics \\ Phillip Colella, Lawrence Berkeley National Laboratory \\ Jeffrey Freidberg, Massachusetts Institute of Technology \\ Jeremy Goodman, Princeton University \\ David Hammer, Cornell University \\ Joseph Hoagland, Tennessee Valley Authority \\ Steve Jardin, Princeton Plasma Physics Laboratory \\ John Lindl, Lawrence Livermore National Laboratory \\ Grant Logan, Lawrence Berkeley National Laboratory \\ Keith Matzen, Sandia National Laboratory \\ Gerald Navratil, Columbia University \\ Arthur Nobile, Los Alamos National Laboratory \\ John Sethian, Naval Research Laboratory \\ John Sheffield, University of Tennessee, Knoxville \\ Mark Tillack, University of California, San Diego \\ Jon Weisheit, Los Alamos National Laboratory
}

\begin{abstract}
This is the final report of a panel set up by the U. S. Department of Energy (DOE) Fusion Energy Sciences Advisory Committee (FESAC) in response to a charge letter from Dr. Ray Orbach (Appendix A). In that letter, Dr. Orbach asked FESAC for "an assessment of the present status of" inertial fusion energy (IFE) research carried out in contributing programs. These programs include the heavy ion (HI) beam, the high average power laser (HAPL), and Z-Pinch drivers and associated technologies, including fast ignition (FI). This report, presented to FESAC on March 29, 2004, and subsequently approved by them (Appendix B), presents FESAC's response to that charge.
\end{abstract}

KEY WORDS: Fusion energy; inertial confinement fusion.

\footnotetext{
This document was prepared as an account of work sponsored by an agency of the United States Government. Neither the United States Government nor the University of California nor any of their employees, makes any warranty, express or implied, or assumes any legal liability or responsibility for the accuracy, completeness, or usefulness of any information, apparatus, product, or process disclosed, or represents that its use would not infringe privately owned rights. Reference herein to any specific commercial product, process, or service by trade name, trademark, manufacturer, or otherwise, does not necessarily constitute or imply its endorsement, recommendation, or favoring by the United States Government or the University of California. The views and opinions of authors expressed herein do not necessarily state or reflect those of the United States Government or the University of California, and shall not be used for advertising or product endorsement purposes
}

This work was performed under the auspices of the U.S. Department of Energy by University of California, Lawrence Livermore National Laboratory under Contract W-7405-Eng-48. 
March 29, 2004

\section{EXECUTIVE SUMMARY}

Igniting fusion fuel in the laboratory remains an alluring goal for two reasons: the desire to study matter under the extreme conditions needed for fusion burn, and the potential of harnessing the energy released as an attractive energy source for mankind.

The inertial confinement approach to fusion involves rapidly compressing a tiny spherical capsule of fuel, initially a few millimeters in radius, to densities and temperatures higher than those in the core of the sun. The ignited plasma is confined solely by its own inertia long enough for a significant fraction of the fuel to burn before the plasma expands, cools down and the fusion reactions are quenched. The potential of this confinement approach as an attractive energy source is being studied in the Inertial Fusion Energy (IFE) program, which is the subject of this report.

A complex set of interrelated requirements for IFE has motivated the study of novel potential solutions. Three types of "drivers" for fuel compression are presently studied: high-averagepower lasers (HAPL), heavy-ion (HI) accelerators, and Z-Pinches. The three main approaches to IFE are based on these drivers, along with the specific type of target (which contains the fuel capsule) and chamber that appear most promising for a particular driver.

In his charge letter (Appendix A) to the chair of the Fusion Energy Sciences Advisory Committee (FESAC), Dr. Orbach, Director of the Office of Science in DOE, noted "the considerable scientific and technical progress" in IFE and asked for "an assessment of the present status of" IFE research carried out in contributing programs. These programs include the HI beam, HAPL, and Z-Pinch drivers and associated technologies, including Fast Ignition. The letter noted that these programs reside in two different parts of DOE, and that both Defense Programs in the National Nuclear Security Agency (NNSA) and the Office of Science "support this review and concur that it be carried out by FESAC."

An IFE Panel was formed to address the charge. The assessment of the quality of the technical work was assigned to those Panel members who are not participants in the IFE program. Overall, they were very impressed by the progress across the program, noting that the three main approaches (HI accelerators, HAPL, and Z-pinch) are at different levels of maturity. The balance between the science and technology emphasis necessarily varies. The recent progress related to these approaches is substantial and the quality of the science and engineering research is excellent. All approaches are currently on track for developing the science and technology to properly evaluate their potential for IFE. However, the planned termination of technology programs in support of the HI approach is not consistent with their importance to HI-IFE, and the Panel is concerned about the impact of this action.

The Panel also examined the potential impact that the Fast Ignition (FI) concept might have on IFE, and observed that each of the approaches to IFE may benefit if the technique of Fast Ignition proves effective. However, since FI is at an early stage of development it would be premature for any of the IFE approaches to rely on the success of FI to achieve an attractive fusion energy system. During the next several years, there is an opportunity to assess the potential of the FI concept utilizing facilities in both Japan and the US (OMEGA, Z, and possibly NIF) through modest OFES investments. 
The primary issues currently facing the IFE program provide the framework for an appropriate set of research plans, which assume continued funding. From this list, the Panel identified the single near-term issue that appears to be the most critical for each of the approaches (HI, HAPL, and ZPinch) and for the FI concept. They are:

HI -- Physics limits to the maximum phase-space density of space-charge-dominated HI beams and the resulting implications for High Energy Density Physics (HEDP) and fusion ignition.

HAPL -- Durability of KrF lasers, and efficiency and beam smoothing in DPSSLs, that will scale to the high-energy requirements for IFE. [The HAPL program is exploring the potential of two types of lasers: the krypton-fluoride $(\mathrm{KrF})$ gas laser, and the diodepumped solid-state laser (DPSSL).]

Z-Pinch -- Physics limitations on power flow in a recyclable transmission line, including the coupling to the pulsed-power driver and the integral target assembly.

FI -- Physics of fuel compression to a uniform-density sphere and of energy transport by relativistic electrons to that high-density fuel to achieve ignition.

These issues, along with many other important basic and applied science issues that form the basis of IFE research plans, must be addressed to assess the potential of IFE. The critical issues related to fuel ignition and burn affect all of the approaches but were not included in the above list because they are being addressed mainly by the Inertial Confinement Fusion (ICF) program in NNSA. The Panel acknowledges this vital role of the ICF program and notes the tremendous leverage that allows the comparatively modest funding for IFE-specific programs to continue to yield important advances. This is a synergistic relationship where IFE research also directly benefits the NNSA mission.

The Panel also found that IFE capabilities have the potential for significantly contributing to HEDP and other areas of science. For example, isochoric heating of substantial volumes to uniform, elevated temperatures should be achievable using HI beams. Investigations of the Fast Ignition concept can lead to exploration of exotic HEDP regimes. Moreover, the rapid turnaround capabilities envisioned for IFE drivers could accelerate progress in HEDP science by enabling a wide community of users to conduct "shot-on-demand" experiments with data rates and volumes far exceeding those obtained on large systems that currently require long times between shots.

In sum, the IFE Panel is of the unanimous opinion that the IFE program is technically excellent and that it contributes in ways that are noteworthy to the ongoing missions of the DOE. Moreover, the Panel agrees with the IFE community that the most efficient way to achieve the ultimate goal of fusion energy is to carry out a coordinated program with some level of research on all of the key components (targets, drivers, and chambers), always keeping the end product and its explicit requirements in mind. The Panel also notes that the scientific and technical challenges posed by IFE, along with their many connections to HEDP, have attracted many outstanding researchers from academia as well as federal laboratories. Success will depend on sustaining the commitment and involvement of such people in a broad spectrum of scientific disciplines. 
March 29, 2004

\section{INTRODUCTION}

\subsection{Background}

Ever since the discovery that thermonuclear fusion is the source of energy that powers our sun and all the stars, there has been the dream to ignite fusion fuel in the laboratory. The interest is driven by two desires: to study matter under the extreme conditions needed for fusion burn, and to explore the potential of harnessing the energy released as an attractive energy source for mankind.

The conditions required to ignite the fuel and sustain a fusion burn include temperatures of about $100,000,000$ degrees or more. The fuel must also be confined and insulated well enough so that energy released from the initial fusion reactions can sustain the fusion process. Achieving those conditions in the laboratory has proved to be a daunting challenge. In stars, the enormous pressure exerted by their gravity leads to these conditions of high temperature and confinement. On earth, the two main laboratory approaches being pursued are magnetic confinement fusion and inertial confinement fusion. Depending on its type and age, a star succeeds in fusing a variety of light nuclei to form heavier nuclei. Attempts to achieve fusion burn in the laboratory will use the fuel that requires the least extreme conditions to create a burning plasma. That fuel is a mixture of deuterium and tritium, two isotopes of hydrogen.

Magnetic Confinement and Inertial Confinement. The magnetic approach uses magnetic fields to confine and insulate the hot fuel, which becomes an ionized gas (a plasma) at the temperatures required for fusion. The inertial approach involves the rapid compression of a tiny spherical capsule of fuel, initially a few millimeters in radius, to densities and temperatures higher than those in the core of the sun. The ignited plasma is confined solely by its own inertia long enough (less than a billionth of a second) for a significant fraction of the fuel to burn before the plasma expands, cools down and the fusion reactions are quenched.

The world scientific community is finally poised to create a burning plasma in the laboratory after more than 50 years of scientific and technological advances. International negotiations are underway to select the site to construct ITER, which will use the magnetic confinement approach. The National Ignition Facility (NIF) is already under construction in the US, and will use the inertial confinement approach. A similar facility, Laser Mega Joule (LMJ), is under construction in France. [Inertial fusion experiments conducted at the Nevada Test Site have demonstrated excellent performance, but NIF is projected to produce the first burning plasmas in a controlled laboratory environment enabling easily repeatable, highly diagnosed studies.]

These facilities will allow the study of burning plasmas under very different conditions involving very different physics. For example, the fusion conditions in NIF will require densities that are about a 100 billion times greater than those in ITER. These NIF experiments will provide highenergy-density-physics (HEDP) conditions never before achievable in the laboratory. The rich scientific opportunities in HEDP are described in a recent National Research Council report [1].

\subsection{Inertial Fusion Energy (IFE)}

The inertial confinement approach has the potential to be an attractive path to fusion energy. That potential is being explored, and these IFE research activities are the subject of this report. 
The motivation for fusion energy research includes the promise of an energy source with no greenhouse gas emissions, and with a virtually inexhaustible fuel supply that is widely available. While these and other features of IFE are attractive, an earlier FESAC report "recognized that difficult scientific and technological questions remain for fusion development." [2] A diversified basic and applied science research portfolio is required to prepare for the realization of the ultimate goal of fusion energy production and to reduce developmental risk. IFE and MFE (magnetic fusion energy) are pursued because they present major opportunities for advancing both science and fusion energy. While they share a common goal, IFE and MFE have significantly different scientific and technological challenges and opportunities. Because of this diversity, the parallel pursuit of IFE and MFE broadens the contributions to science while reducing developmental risk.

Conceptually, IFE can be harnessed to generate electricity and other useful products from a steady sequence of inertial confinement fusion (ICF) events. Each ICF event involves placing a small capsule of fuel in a chamber and then compressing and heating it to ignition by some type of "driver" that generates intense pressure on the outside of the capsule. Fusion-power system studies indicate that the energy released per event could range between hundreds of megajoules and several gigajoules. The corresponding repetition rates would range from several per second to about once every ten seconds for a 1-GW(e) power plant.

Four principal technical requirements must be achieved in a cost-competitive, environmentally attractive manner for IFE to be successful:

1) High Energy Gain and Efficiency: The efficiency of the driver in converting energy from the electrical power grid to the energy needed to compress the capsule, coupled with the energy "gain" of the capsule (ratio of energy released to the energy needed to compress and heat the fuel) must be sufficient to yield substantial net energy.

2) Repetition Rate: The driver, target (which includes the fuel capsule) fabrication, and reaction chamber must operate at a repetition rate that is sufficient to produce economically useful power. The chamber must be restored to a sufficiently quiescent state after each shot to allow insertion of the next target, and for the transmission and focusing of the next pulse of energy from the driver to that target.

3) Energy Conversion and Tritium Breeding: The energy released from the burning deuteriumtritium (DT) fuel is mainly in the form of energetic ions, neutrons, and x rays. This energy must be absorbed by the chamber and converted into "high-grade" thermal energy that can be efficiently used to drive electric generators. The chamber must also utilize the emitted neutrons to breed sufficient new tritium (from lithium) to sustain the fuel supply.

4) Durability: The components in an IFE system must carry out the above functions with sufficient durability for the high capacity factors required in an attractive energy system.

Approaches to IFE. This complex set of interrelated requirements for IFE has motivated the study of novel potential solutions. Three types of "drivers" for fuel compression are presently studied: high-average-power lasers (HAPL), heavy-ion (HI) accelerators, and Z-Pinches. The need for efficient coupling of energy from these drivers to the capsule has motivated different conceptual designs for the "targets," which contain the capsule of fuel.

In broad terms the targets can be categorized as "direct drive" or "indirect drive" types. Both types of targets have been used in the ICF program for various physics studies. Fusion capsules 
for both target types are conceptually similar, and are envisaged to consist of a tiny, spherical, cryogenic solid shell of DT fuel or DT-wetted foam, coated with plastic or other materials. In the case of direct drive, the driver beams (e.g., lasers) are focused directly on the surface of this coating. For indirect drive, the driver energy is delivered to the interior of a high- $Z$ (heavy element) enclosure (hohlraum) so that the material at or near the inside surface of the hohlraum is heated to 2 to 3 million degrees. This creates blackbody $x$-ray radiation that impinges on the capsule at the center of the hohlraum.

The impinging energy (directly from driver beams or from x-rays) heats up the coating on the outer surface of the capsule. The heated coating ablates radially outward, generating an inward momentum impulse very much like a standard rocket engine. Driven by this "rocket" the shell implodes, reaching high velocities, and then slows down as the pressure builds up in the fuel. As the shell slows down, its kinetic energy is converted into internal energy (i.e. pressure) of the material enclosed by the imploding shell. The rapidly converging fuel creates a "hot spot" at the center that reaches its maximum temperature and pressure when the shell stagnates. At pressures of hundreds of gigabars and a temperature of about $10 \mathrm{keV}$ the hot spot ignites and a fusion burn front propagates outward through the bulk of the compressed fuel. [Plasma temperatures are frequently measured in units of electron volts $(\mathrm{eV})$ or thousands of electron volts $(\mathrm{keV})$. Since 1 $\mathrm{eV}=11,600^{\circ} \mathrm{K}$, then $10 \mathrm{keV}$ is slightly more than 100 million degrees.]

System studies indicate that each driver type is best matched with a specific target design and to a specific reaction chamber (including energy conversion) design. Two general types of chambers are being examined to accommodate the specific needs of the different drivers and targets. Drywall chambers have armor to withstand the energetic radiation and debris from the targets. The thick liquid-wall chambers have the advantage of continually replacing the surface exposed to this radiation and debris but with the added complexity of managing the flowing liquid. The three main driver types (HAPL, HI, and Z-Pinch), coupled with their corresponding target and chamber types, become the three main approaches to IFE. The following paragraphs briefly describe the present concepts for these three approaches.

High-Average-Power Lasers. The HAPL approach involves research on both kryptonfluoride $(\mathrm{KrF})$ gas lasers and diode-pumped solid-state lasers (DPSSL). The main-line approach for either laser is to use direct-drive targets to maximize energy coupling efficiency to the capsule. Advances in laser techniques indicate that the spherical uniformity of illumination required by these targets may be achievable. Dry-wall chambers for energy conversion appear to be most compatible with the final optics and the penetrations in the chamber wall needed for spherical illumination. Dry walls are also compatible with the relatively low x-ray output from direct-drive targets, as compared with that from indirect-drive targets.

Heavy-Ion Accelerators. The HI-accelerator approach plans on indirect-drive targets because the large ICF target physics database for indirect drive allows good definition of HI beam requirements, and because two-sided illumination is compatible with thick liquid protected chambers. The loss in efficiency compared to direct drive should be offset by the higher efficiency that is expected from HI accelerators. Liquid wall techniques for absorbing the energy while protecting structural material appear usable with the HI approach due to geometrically limited wall penetrations (spherical illumination not needed) and less demanding requirements for protecting final focusing optics from chamber and target debris compared with lasers.

Z-Pinches. The Z-Pinch approach produces $\mathrm{x}$-rays from an imploding cylindrical array of current-carrying wires that stagnate on a low-density-foam cylinder. One or two of these assemblies provide the $\mathrm{x}$-rays inside of a hohlraum for the indirect-drive-target design. The high 
$\mathrm{x}$-ray-generation efficiency of the pulsed-power driver and the z-pinch should compensate for the lower efficiency of this indirect-drive target design. The Z-Pinch driver, in contrast with the other two IFE approaches, is physically connected to the target by transmission lines. Lower repetition rates than HAPL or HI, and hence higher fusion yields per target, are envisioned to allow for replacing the transmission lines on each shot. Liquid walls are the baseline chamber approach, as in the HI approach, and they should be useful in dealing with the larger fusion yields.

The development of high-energy petawatt $\left(10^{15}\right.$ Watts) lasers raises the possibility of reducing the compression-driver requirements for any of the three approaches. The potential improvement relies on the extremely short pulse ( $\sim 10 \mathrm{ps})$ of a petawatt laser to heat and ignite a small portion of the fuel near the edge of a compressed capsule. This "Fast Ignition" concept separates the functions of compression and ignition, and potentially relaxes the constraints on compressiondriver energy and target uniformity because no central hot spot is required. This flexibility may allow higher-gain capsules, and simplification of chamber and driver specifications.

\subsection{IFE and Inertial Confinement Fusion (ICF) Programs}

The IFE program in the US exists in the context of both weapons and energy research. The DOE National Nuclear Security Agency (NNSA) has a major program in ICF because of its relevance to the nuclear weapons Stockpile Stewardship Program (SSP). The ICF program, funded at $\$ 504 \mathrm{M}$ in FY03, supports major facilities including the 1.8-MJ NIF laser $(\$ 290 \mathrm{M})$ being constructed at the Lawrence Livermore National Laboratory, the 30-kJ OMEGA laser facility at the University of Rochester, the 2-MJ Z pulsed-power facility at Sandia National Laboratories, and the 3-kJ Nike laser at the Naval Research Laboratory. [The energies listed represent the energy that each facility can deliver to a target.] These facilities and the program more generally devote a major fraction of their resources toward SSP research that is much broader than just achieving ignition, and that includes a variety of high-energy-density physics (HEDP) topics.

Decades of experimental, theoretical, and computational R\&D carried out in the ICF program have led to an understanding of the science and technology needed for single-shot ignition and burn. Much of this information is also essential to the IFE program. Relevant topics include driver-energy absorption by the capsule, driver asymmetries, capsule nonuniformities, implosion hydrodynamic stability, hot-spot ignition, and burn propagation. The ICF program has the goal of achieving ignition on NIF within the next decade. The close interactions between the ICF and IFE programs, particularly in the area of target physics, are essential to the success of the much smaller IFE program.

IFE research programs currently exist in both NNSA and the Office of Fusion Energy Sciences (OFES) within the DOE Office of Science. Congress has added significant funding to the NNSA budget for IFE during the past five years, including \$25M in Fiscal Year 2004, to support research through the High Average Power Laser (HAPL) program. The HAPL program is focused on developing the science and technology for the laser drivers and other components needed for fusion energy production, and is closely coupled with the target physics work carried out in the ICF program. In FY04 Congress also added \$4M to explore the Z-Pinch as an IFE driver. These efforts are focused on the goals and needs of IFE that are beyond those of ICF.

The OFES component of the IFE program is focusing mainly on the scientific underpinnings of the Heavy-Ion (HI) approach. This strategy emphasizes research topics such as non-neutral plasmas and accelerator physics issues essential for the HI approach and relevant to other fields 
of science. Indeed, because of the potential for IFE research to contribute to the field of HEDP, OFES has adopted the following performance measure for its IFE program [3]: "High Energy Density Physics/IFE: Progress in developing the fundamental understanding and predictability of high energy density plasma physics, including potential energy producing applications." The OFES has also sponsored research in the areas of target design and fabrication, Fast Ignition, HI chambers, and system studies for HI and lasers. In FY03 the OFES budget for all IFE activities was about $\$ 17 \mathrm{M}$. The FY04 appropriations provided only $\$ 15 \mathrm{M}$ for IFE, including only closeout funds for technology. Systems studies were eliminated. The closing out of these programs is reflected in Table 1, which summarizes the funding source for various activities important to the IFE program.

Table 1

Source of Funding for IFE-Relevant Activities

\begin{tabular}{|l|c|c|c|}
\hline & NNSA Program & OFES Program & Report Section \\
\hline HEAVY-ION BEAMS & & & \\
\hline Driver Science & & OFES-IFE & 3.2 .1 \\
\hline Final Optics (magnets) & & & 3.2 .1 \\
\hline Target Physics & & OFES-IFE & 3.1 .1 \\
\hline Target Fabrication & & & 3.1 .4 \\
\hline Target Injection & & & 3.1 .4 \\
\hline Chamber & & & 3.4 .1 \\
\hline LASERS & HAPL & & \\
\hline Driver Science/Technology & HAPL & & $3.2 .2,3.2 .3$ \\
\hline Final Optics (mirrors) & ICF (+HAPL) & & 3.2 .3 \\
\hline Target Physics & HAPL (+ICF) & & 3.1 .2 \\
\hline Target Fabrication & HAPL & & 3.1 .4 \\
\hline Target Injection & HAPL & & 3.1 .4 \\
\hline Chamber & & & 3.4 .1 \\
\hline Z-PINCHES & Z-IFE & & 3.2 .4 \\
\hline Driver Science/Technology & Z-IFE & & 3.2 .4 \\
\hline Recyclable Transmission Line & ICF & & 3.1 .1 \\
\hline Target Physics & ICF (+Z-IFE) & & 3.1 .4 \\
\hline Target Fabrication & Z-IFE & & 3.4 .1 \\
\hline Chamber & & & 3.1 \\
\hline FAST IGNITION & ICF & & 3.1 .4 \\
\hline Petawatt Lasers & & OFES-IFE & \\
\hline Target Physics & & & \\
\hline Target Fabrication & & \\
\hline
\end{tabular}

Since the distinction between the IFE and ICF programs is essential for the clarity of this report, the following descriptions are provided for the reader's convenience:

IFE program -- the DOE-sponsored research in inertial fusion energy, including the OFES research in Heavy-Ion Beams and Fast Ignition, and the NNSA HAPL and Z-Pinch-IFE programs.

ICF program -- the NNSA-funded program in inertial confinement fusion, excluding the HAPL and the Z-Pinch-IFE programs. 
Only a few countries have major research programs in inertial fusion. These include Japan and France, with the US program being the largest. The Japanese program is exclusively devoted to energy research. The emphasis is on Fast Ignition using the 12-beam $~ 10-\mathrm{kJ}$ GEKKO laser and a $0.4-\mathrm{kJ}$ petawatt laser. The work is carried out at the Institute for Laser Engineering at Osaka University, where the FIREX1 construction project is adding a $\sim 10-\mathrm{kJ}, 10-\mathrm{ps}$, 4-aperture laser to GEKKO for Fast Ignition research. The French program is mainly oriented toward weapons research and HEDP. Facilities include a 1-kJ petawatt laser capability at LULI (Ecole Polytechnique) in Paris and the 60-kJ LIL facility at CESTA near Bordeaux. As mentioned earlier, the LMJ facility is under construction and will have an energy level comparable to NIF. It also has a similar schedule for reaching ignition.

\subsection{Formation and Activities of FESAC's IFE Panel}

Charge to FESAC. In his charge letter (Appendix A) to the chair of the Fusion Energy Sciences Advisory Committee (FESAC), Dr. Orbach, Director of the Office of Science in DOE, noted "the considerable scientific and technical progress" in IFE and asked for "an assessment of the present status of" IFE research carried out in contributing programs. These programs include the HIbeam, HAPL, and Z-Pinch drivers and associated technologies, including Fast Ignition. The letter noted that these programs reside in two different parts of DOE, and that both Defense Programs in NNSA and the Office of Science "support this review and concur that it be carried out by FESAC."

IFE Panel Organization and Activities. FESAC's IFE Panel membership included 7 members of FESAC and 13 additional experts. Ten of the members are experts associated with IFE programs and the other ten are not, but have expertise in MFE, HEDP, and other relevant disciplines.

The IFE experts were assigned the responsibility of ensuring that all of the relevant information about the status of the programs, including recent advances, was made available to the Panel through presentations and written material. They were also responsible for explaining the major issues facing the programs and identifying possible approaches to resolving those issues. In addition, they were responsible for providing information about how IFE research has or might contribute to other scientific endeavors, including HEDP.

The members who were not participants in the IFE program were assigned the responsibility of assessing the quality of the IFE research and reviewing the key issues facing the program. They were also to comment on the appropriateness and timelines of possible research activities to resolve the issues, taking into account the availability of resources and appropriate facilities, and the value of the advances in technical understanding that might accrue. They were also assigned the task of evaluating the potential impact of the IFE programs on HEDP and other areas of science.

The Panel began its work by creating a reading list, which included recent reviews of the IFE program. The Panel met twice and held 5 formal conference calls. The meetings involved researchers from the IFE community in addition to Panel members. Representatives of the US government and one IFE expert from Japan were among those who interacted with the Panel. The Panel extensively used a website and email to share information between meetings and during conference calls. 
The FESAC Priorities Panel sought input from the IFE Panel on key issues that motivate MFE and IFE research, as expressed in a list of Overarching Questions and Topical Questions. The IFE Panel response was provided in a letter and a subsequent email.

Outline of Report. First, the report describes a few general observations and findings that grew out of the Panel's many discussions. Second, the report provides assessments of the technical progress in IFE, and of the research quality. Third, it describes the critical scientific issues for the IFE program, and evaluates the readiness of the program to embark on activities that will lead to new advances in understanding toward the goal of fusion power. Fourth, the report considers potential contributions from IFE programs to HEDP, the ICF program, and other areas of research.

The report responds to the following four topics quoted from the charge letter:

1. The current status of the scientific basis and related technology of each of the approaches to IFE, including an assessment of the quality of work being carried out in the programs. [See Section 3]

2. Critical scientific issues identified in each of the approaches to IFE that would contribute to understanding the long-range potential of IFE. [See Sections 3 and 4]

3. The impact that fast ignition as a concept improvement may have on IFE. [See Sections 3 and 4$]$

4. The potential contribution of the various IFE program elements to the emerging field of High Energy Density Physics. [See Section 5] 


\section{SOME GENERAL OBSERVATIONS AND FINDINGS}

The Panel found it challenging to attempt a coherent and effective evaluation of IFE when the activities funded through OFES and NNSA differ both in their near-term strategies and in their technical approaches to achieving fusion energy. The OFES-funded IFE activities have been asked to emphasize a subset of the science relevant to IFE, with particular emphasis on areas relevant to HEDP. The NNSA-funded HAPL and Z-Pinch IFE activities take an integrated approach to developing the key science and technologies together as a coherent system needed for fusion energy. Each program also pursues technical approaches that differ greatly in their scientific and technology issues. The set of general observations and findings in this section derive from the process of working through this assignment.

These findings are listed here to set the context for the rest of the report. More specific findings, including those that respond to the itemized topics in the charge letter, are found within the text of Sections 3 through 5 .

Finding: The Panel recognizes and respects the reasons for the differences in near-term focus of OFES and NNSA sponsored programs. Although near-term strategies differ, the ultimate goal of all IFE research is fusion energy production. The long-term potential for fusion power provides an exciting and unifying purpose for all IFE research activities.

Each of the approaches to IFE (see Table 1) involves basic and applied scientific challenges. These challenges are described in Sections 3 and 4. The scientific topics include accelerator physics, equations of state in the HEDP regime, plasma chemistry, laser-plasma interactions, the material science underpinning optical damage at high intensities, physics limits of power flow in transmission lines, and energy absorption in materials (chamber walls) that reach HEDP intensities, to name just some.

Finding: IFE research involves a rich set of scientific challenges. Substantial advances in a spectrum of scientific disciplines will be required to effectively assess the long-term potential of IFE. Many outstanding researchers from academia as well as federal laboratories are pursuing a range of exciting IFE science topics.

The IFE program needs to address the scientific and technical issues associated with the complex interrelated requirements for targets, drivers, and chambers that pose the greatest uncertainty in assessing the ultimate potential of IFE. As the Panel struggled with selecting the most critical near-term issues identified in Section 4, it was guided by the ultimate goal of IFE and by the present understanding of the various elements of an energy producing system.

Finding: Understanding the interrelated scientific and technological issues of the key components of IFE within the framework of an integrated system is an essential input for prioritizing IFE research activities, whether for the science-focused OFES program or for the NNSA program. Careful prioritization is particularly important given the limited resources available to these IFE activities.

The prevailing view in the IFE Panel is that the most efficient way to achieve the ultimate goal of fusion energy is to carry out a coordinated program with some level of research carried out on all of the key components (targets, drivers, and chambers), keeping the requirements of the end product in mind. However, the Panel members had widely varying views on the relative levels of effort among the components and the degree of focus on science or technology that would 
provide the most efficient development path, given the present state of understanding. Keeping in mind this broad description of a coordinated program, the Panel supports the following finding.

Finding: Carrying out a coordinated IFE research program allows a more efficient approach for developing a fundamental understanding of the science that is necessary for IFE.

An obvious challenge for IFE is the long timescale that is projected, given present funding levels, to resolve the interrelated scientific and technical issues and to demonstrate the potential of IFE. This challenge requires the continuing recruitment, training, and retention of outstanding young people.

Finding: The scientific and technical challenges posed by IFE, along with their many connections to HEDP, and the grand ultimate purpose of fusion power highlight both the need and the opportunity to attract outstanding researchers for future success. In order to identify and exploit key opportunities and synergies with HEDP and other exciting topics, improved coordination is needed between various scientific communities. The series of workshops on laboratory astrophysics with lasers is a model that could be emulated. 


\section{ASSESSMENT OF THE TECHNICAL STATUS -- RECENT PROGRESS AND CHALLENGES}

Given the large number of possible combinations of drivers, targets, and chambers, it is prudent to focus most of the research resources on the combinations that appear to be most promising. As described in the Introduction, the HAPL program emphasizes direct-drive targets and dry-wall chambers. The HI accelerator and Z-Pinch efforts emphasize indirect-drive targets and thick liquid-wall protection. Fast Ignition may be compatible with both target/chamber combinations and any of the compression drivers.

This section contains an overview of the recent advances made on these three main approaches to IFE and on the Fast Ignition concept. The primary remaining issues for each topic are noted along with the general plans of the program to address them. A brief assessment of the quality and appropriateness of the work in each of these areas is also provided. In carrying out this assessment, the members of the Panel who are outside of the IFE program examined the achievements in light of the goals that were established for the various program elements in recent years $[4,5,6]$ while taking into account the availability of funds since the goals were established. Additional factors in the assessment process included the presentations given to the panel by IFE experts, the publication record of the researchers, including the level of exposure in national and international meetings, and the identification of the remaining critical issues with defined research programs to resolve them.

\subsection{Targets}

Although targets being evaluated for IFE can be broadly categorized as either indirect drive or direct drive, target details can vary substantially depending on the choice of driver and chamber. Figure 1 illustrates some of this diversity.

For IFE, a key parameter in matching a target with a driver is the target gain, G. G must exceed a certain minimum that depends on the fusion system's driver efficiency. The inverse of the product of $\eta \mathrm{G} \varepsilon$ is the fraction of the electric energy produced that is used to power the driver, where $\eta$ is the driver efficiency and $\varepsilon$ is the overall efficiency of conversion of thermonuclear energy to electricity. For example, for $\varepsilon=50 \%$ and a driver efficiency $\eta=10 \%$, a target gain of 100 would result in $20 \%$ of the electric output being used to power the driver. In general, indirect-drive targets have lower gains than direct-drive targets, and therefore require higher efficiency drivers. Indirect-drive targets are also more complex, but they impose less stringent requirements on the focusing and uniformity of driver energy delivered to the target.

The Fast Ignition (FI) concept uses one of the drivers (HI, HAPL, or Z-Pinch) to compress the target shown in Figure $1 \mathrm{~g}$, and then uses an extremely intense $\left(10^{19}-10^{20} \mathrm{~W} / \mathrm{cm}^{2}\right)$ laser beam to ignite a propagating thermonuclear burn wave in the compressed, but cold, target. This concept has the potential of increasing target gain and/or easing the detailed requirements on compressordriver energy delivery to the target. The concept is at an early stage of investigation, the physics is complex, and the technology is challenging. The potential and the challenges are described in Section 3.3. 

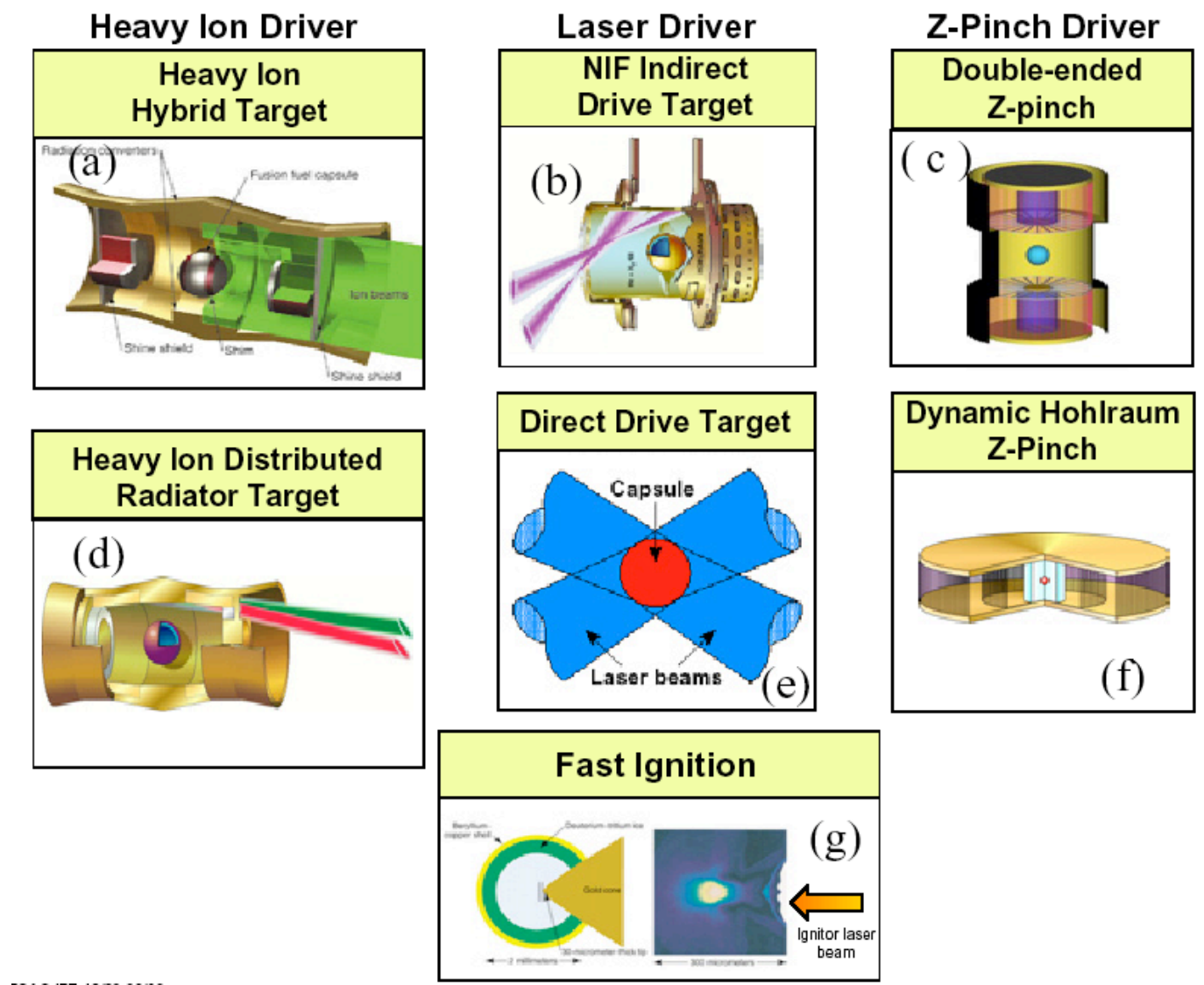

Figure 1. A wide range of targets is being examined for IFE.

\subsubsection{Indirect Drive}

The major differences between laser (NIF), HI, and Z-Pinch indirect drive targets are related to the physics issues of $\mathrm{x}$-ray production and the detailed hohlraum geometry utilized to achieve symmetry of the radiation driving the implosion. Capsule physics is essentially independent of the source of x-rays. Also, the physics of x-ray transport within the various hohlraum concepts as well as of hohlraum energetics is common to all indirect-drive targets. Therefore, OMEGA, Z, and NIF can investigate implosion and ignition issues for all of the indirect-drive targets being considered for IFE. Similarly, IFE target innovations and insights can, and have been, incorporated into the ICF program.

\section{Indirect Drive with Lasers (NIF)}

This is the baseline approach to ignition on NIF (Figure 1b). OFES supports a small amount of IFE target design work on techniques that could simplify the laser-beam geometry relative to that utilized on NIF. Essentially all other target design work for laser indirect drive is supported by the ICF Program and is specifically aimed at improving the performance of NIF. For example, hohlraums are being designed with material mixtures that reduce x-ray losses into the wall, and with smaller laser entrance holes. These improvements are expected to enable reduced hohlraum sizes, increased coupling efficiency to the capsule, and higher gain. There are now indirect-drive 
target designs for NIF with gains up to 50. These advances have positive implications for IFE. For NIF, the major challenge is to control laser-plasma interaction (LPI) effects with only a modest $(10-20 \%)$ energy penalty.

\section{Indirect Drive with Heavy-Ion Beams}

Indirect drive is the baseline target concept for the HI-beam approach, which will benefit from the ignition target physics studies being planned for NIF. In addition, HI drivers are expected to have an efficiency of $25-40 \%$, so relatively low target gains (25 to 50) are projected to be adequate for HI IFE.

All recent HI-driven target design work has been funded by OFES. Since beam focusing to a small spot size is a challenge for HI beams, this target research included a systematic study of the tradeoffs among drive symmetry, beam spot size, and capsule hydrodynamic stability. Two designs of interest resulted. One more efficiently utilizes driver energy but requires a more challenging (1-2 mm radius) ion beam spot on target (Figure 1d), and the other greatly relaxes the beam radius requirement ( $\sim 5 \mathrm{~mm}$ radius), but $\sim 20 \%$ more delivered beam energy is required (Figure 1a). It is noteworthy that optimization of capsule performance of HI-driven targets has had a substantial impact on the optimization of capsules for NIF targets. At present, the most critical issue for HI fusion is to better understand the physics that may limit beam brightness on the target.

\section{Indirect Drive with Z-Pinches}

Recent breakthroughs in efficiently producing intense, $z$-pinch-driven, thermal $x$-ray sources and achieving symmetric capsule implosions on the $\mathrm{Z}$ facility have created a new path for achieving indirect-drive ICF. This research is supported by the NNSA ICF program as part of a broadbased effort to evaluate options for targets with yields of greater than 500 MJ. The "doubleended z-pinch hohlraum" configuration (Figure 1c) and the "dynamic hohlraum" (Figure 1f) configuration have both demonstrated x-ray production with about $15 \%$ overall electrical efficiency when a cylindrical, radially imploding z-pinch plasma stagnates on a low-density foam cylinder. Since $\mathrm{x}$-ray production efficiencies of up to $25 \%$ are feasible, IFE target gains in the range of 50 or more are required.

The DT-fuel-capsule location is quite different in the two cases. In the double-ended z-pinch target, the capsule is in a static hohlraum. A decade of experiments has demonstrated control of radiation symmetry to within a factor of two of that required for high-yield capsule implosions. Further advances are needed in the scaling of $\mathrm{x}$-ray power and pulse shape as the current is increased from $20 \mathrm{MA}$ to the $\sim 60 \mathrm{MA}$ required for high yields.

The dynamic-hohlraum concept embeds the spherical fusion capsule inside the low-density foam cylinder that is centered in the single high- $Z$ wire array. This arrangement offers the potential for about twice the efficiency in delivering energy to the capsule, compared with the double-ended zpinch target. However, the physics issues associated with achieving a highly symmetric capsule implosion are closely coupled to the physics of the z-pinch implosion itself.

While the target designs take advantage of much of the capsule physics developed in the laser ICF program, the capability to perform fully integrated $2 \mathrm{D}$ and $3 \mathrm{D}$ calculations that include the physics of the wire arrays is still being developed. 
March 29, 2004

\subsubsection{Direct Drive with Lasers}

Direct-drive targets (Figure 1e) are conceptually simpler than indirect drive targets and they have higher overall energy-coupling efficiency to the fuel capsule. Direct-drive target design has been supported entirely through the ICF and HAPL programs of NNSA.

The efficiencies of laser drivers ( $\mathrm{KrF}$ and DPSSL) are projected to be about $7 \%$ percent. At these values, target gains of at least 120 are needed for IFE. Recent work predicts capsule gains of 120-170 for a few-MJ driver.

The major challenge in realizing sufficient gain from a direct-drive target is suppression of hydrodynamic (Rayleigh-Taylor) instabilities, which can be seeded by the non-uniformities of either the capsule surface or the driver-beam illumination. Experiments have shown that adding a thin high-Z layer (such as Pd) on the surface of the target substantially reduces the imprint of laser non-uniformities, and hence mitigates the seeding of hydrodynamic instabilities. In addition, the energy-deposition scale length in the ablator is much shorter for direct drive than for indirect drive. For laser beams impinging directly on the ablator, the scale length is determined by electron thermal conduction; for indirect drive, the scale-length is set by the more deeply penetrating x-rays. However, by tailoring the post-shock entropy profile of the ablator, calculated instabilities in direct-drive targets can be reduced to levels comparable to those seen in indirectdrive targets, while still maintaining high gain. The major experimental challenge now is to see whether instabilities can actually be controlled to the level that is predicted to be needed for achieving ignition and burn.

Obtaining optimal gain in direct-drive targets is anticipated to require a beam-pointing accuracy that is greater than that for indirect drive. Determining the pointing accuracy requirements, and achieving them under IFE conditions where targets are rapidly injected into a chamber, is the subject of ongoing research.

Assessment Analysis: Progress toward understanding the issues that affect the gain in IFE target designs was the metric used by the Panel in assessing the target physics work. Noteworthy accomplishments are: (1) the much improved understanding of the trade-offs (among drive symmetry, beam spot size, and capsule hydrodynamic stability) in the design of high-gain, HI indirect-drive targets; and (2) the discovery that instabilities in direct-drive targets can be suppressed by thin, high- $Z$ capsule coatings, as well as by tailoring the ablator's post-shock entropy profile, and hence its density profile.

Finding: The combined IFE plus ICF target physics research portfolio is appropriately diverse, and the technical work is of very high quality. Excellent progress involving one or more of the key performance criteria - implosion symmetry, capsule stability, and high gain - continues to be made on central hot-spot targets being designed for the various driver schemes.

\subsubsection{Theory and Simulation}

NNSA-funded efforts in ICF and IFE have developed a variety of computer codes to simulate the performance of inertial fusion targets. These include the 3D radiation hydrodynamics codes HYDRA (developed at LLNL) and FAST3D (developed at NRL), as well as a number of 1D and 2D codes. These codes have all of the physics packages needed to simulate a broad range of problems in capsule design, and in hohlraum design for indirect drive. In addition, the investments made by NNSA through its Advanced Strategic Computing Initiative (ASCI) have markedly improved many of the computational tools, as well as dramatically increased the 
available computing power. Another important component of the success of simulation is a robust program of validation of the codes against experiments performed at Nike, OMEGA, Z, and NIF. As a consequence of these investments, simulation has become an important tool for target design, leading to improvements in robustness and predicted yield.

There are a number of areas in which advances in simulation capability are needed by the IFE program. Uncertainties in material properties such as opacities of high-Z materials and some equations of state impose fundamental limitations on the a-priori accuracy of calculations of the performance of targets designed for ignition and burn. There is an ongoing ICF research program to reduce these uncertainties and to validate material models currently in simulation codes. (As an example, the compressibility of hydrogen in the megabar range, based on several recent experiments, is uncertain to $50 \%$. This, in turn, leads to an uncertainty in the precise pulse shape required for a low-entropy implosion.) In addition, in some areas, improvements in simulation capability are needed to obtain higher-fidelity computations. These include modeling such physical processes as laser-plasma instabilities and magnetohydrodynamic effects, as well as new computational capabilities such as adaptive meshing and more accurate numerical modeling of material interfaces. These advances will increase the range of length scales that can be represented in a single calculation. Such algorithmic improvements are particularly important for simulating the growth of interface instabilities, for which nonlinear multimode effects are important. All of the developments mentioned here will be of critical importance to the success of the Fast Ignition concept, because of the higher energy densities, the broader range of scale lengths, and the multidimensionality of the geometry.

The impact of the NNSA-funded simulation efforts on IFE research is quite substantial. The investments in code capabilities, material models, and experimental validation at ICF facilities all are applicable to support the resolution of corresponding problems in IFE. Furthermore, simulation is the bridge between IFE and HEDP science: simulation codes and models that are bench-marked using IFE experiments can then be used to investigate the corresponding phenomena in areas such as astrophysics, for which controlled experiments are not possible. However, since many of the NNSA-developed codes are not widely available to university researchers, theoretical collaboration with some IFE communities can be significantly limited.

Assessment Analysis: The Panel's metric for measuring the success of simulation in IFE target design is the extent to which simulation is an integral tool to the target design process and quantitative analysis of experiments. Such a successful use of simulation in science is difficult to achieve, and consequently represents an outstanding accomplishment. Of particular note for laser indirect drive targets, a variety of 1D, 2D, and 3D codes have been used to improve the design of all aspects of the capsule, including the effect of the shape of the laser pulse, of the composition of the ablator, and of the composition and thickness of the high- $Z$ shell on the growth of instabilities and therefore the overall gain.

Finding: Simulation has a substantial impact on enhancing the performance and understanding of existing and advanced target concepts. The ICF and IFE programs have developed valuable capabilities for driver-matter interactions, including an extensive validation program against experiments performed at NIKE, Z, and OMEGA. Nonetheless, new simulation capabilities are needed, particularly in the areas of material models, laser-plasma interactions, and in the range of scales that can be represented. The IFE program would also benefit from developing an opensource simulation capability in the area of capsule physics, particularly in connection with HEDP. 


\subsubsection{Target Fabrication}

Credibility of the IFE program depends upon a viable fabrication plan for the large quantities of targets (100,000 to 500,000 per day for a 1000-MW(e) plant, depending on the target yields being considered) that will have to be made for a fraction of a dollar per target. In recent decades, the ICF program has developed and used a wide range of techniques to generate spherical, cylindrical and planar laser and z-pinch targets in small numbers for room temperature as well as cryogenic experiments. Robust processes are being developed that are capable of producing large quantities of stringently specified, but virtually identical targets and delivering them to the center of a target chamber with high accuracy. Significant IFE advances have been made in capsules, DT-fuel filling and layering, hohlraum production, and target injection, with potential benefits also accruing back to ICF target activities (see Section 5.2).

Processes needed for supplying targets for an IFE power plant have been identified, and the critical issues are understood. Figure 2 shows a potential target fabrication sequence for direct drive (HAPL) and indirect drive (HI). Capsules are fabricated, filled with DT and cooled to near the DT triple point where a DT ice layer is formed. The layered capsules are loaded into a hohlraum or a sabot, injected into the chamber, and tracked in flight to provide data for the final beam steering. The current status and future challenges associated with these target supply steps, and extensions to Z-Pinch drivers and Fast Ignition, are briefly described in this Section.

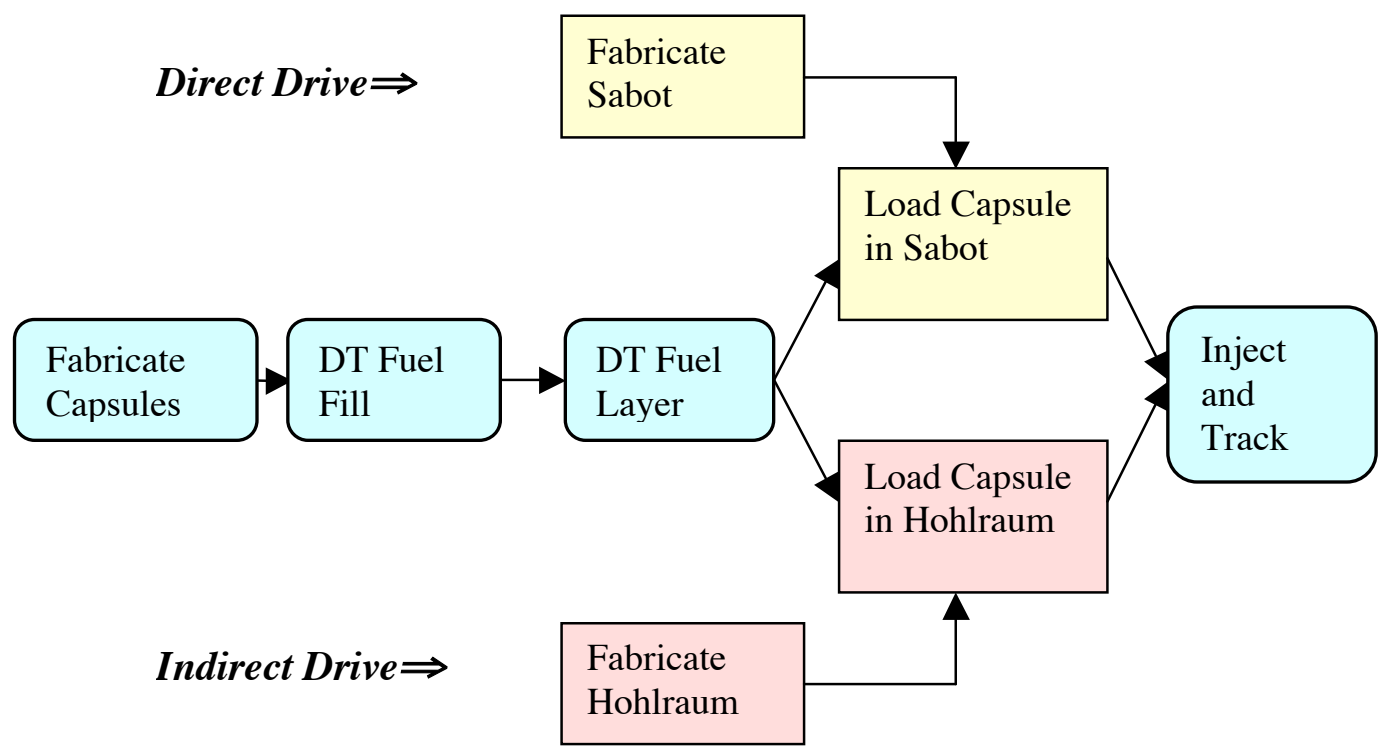

Figure 2. Likely target fabrication sequence for IFE targets.

By leveraging the ICF program technological base, IFE feasibility and proof-of-principle target development efforts have made progress in several areas. In the case of the HAPL targets [7], the feasibility of producing foam capsules of appropriate dimensions and density on a batch production basis has been demonstrated. Further work is needed to improve capsule quality, 
reproducibility and large-scale production. Methods have been demonstrated to apply the overcoat to the foams and apply a DT-permeable, IR-reflecting layer outside the target. Modeling of the DT-fuel filling process has indicated that capsule filling systems can operate with acceptably low $(<1 \mathrm{~kg})$ tritium inventories. Other initial steps have been taken to address the open questions concerning the feasibility of high-rep-rate fusion-target manufacture and injection into a chamber, such as DT-ice-layer formation in foams; and layering of multiple targets in a fluidized bed (using a surrogate fuel material at room temperature to simulate DT ice). In the case of $\mathrm{HI}$ targets, hohlraum production using advanced fabrication processes like those used for micro- and nano-electronics component fabrication has been identified, and experiments to demonstrate the use of laser chemical-vapor deposition as a means of producing low-density metal foams are underway.

Each of the individual processes needed for ignition-target fabrication has been conceptualized or demonstrated at some level, but neither direct-drive nor indirect-drive targets have yet been fabricated that meet the stringent specifications needed to achieve ignition, even for single-shot ICF. Although the integration of these processes to produce the required targets has not yet been demonstrated, there are no barriers known at this time that should prevent success.

Targets for Z-Pinches are also being investigated conceptually, and Fast-Ignition targets and target production processes are being scoped for a range of compression drivers. The HAPL program has built a target injection facility and used it to accelerate surrogate IFE direct-drive targets to the proper velocity, and to demonstrate the concept of the separable sabot. These tests have been done both single-shot and in 3-shot bursts. The facility could also be used to study injection of HI and FI targets. An upgrade for rep-rated use with cryogenic targets should be completed in the future that eventually could accelerate mass-produced targets that meet fusion power requirements. These should be injected into a high temperature test chamber to demonstrate survival and target tracking in the hostile environment during injection. The target will be shot with a low-energy pulsed laser beam in flight, all at full operating rates of about 6 Hz. A suite of target characterization and in-flight diagnostics will be used to verify the achievement of all aspects of the process.

Assessment Analysis: The metric used by the Panel to assess target fabrication efforts was the progress toward understanding how to mass-produce cost-effective high-yield IFE targets and how to deliver them, as needed, to a fusion chamber. To this end, the individual processes required for fabrication of laser- or HI-beam-driven targets have all been identified and studied individually at some level. Additionally, there are efforts to improve Z-Pinch- and FI-targetfabrication concepts, which are in the early stages of development. Very promising target injection experiments are now underway at a new, versatile facility. The Panel noted that there has been substantive progress concerning each of the key fabrication and injection issues, and moreover that no barriers to the eventual, successful integration of the relevant processes have been identified.

Finding: Substantial progress in target fabrication has been made, by using a combination of modeling and experiments with surrogate materials, as well as by leveraging the ICF program knowledge base. The work in this area has been of high quality. Current-day targets do not meet all ignition specifications, but concepts for producing practical IFE targets have been developed and there is a plan to demonstrate these concepts. However, many difficult questions have yet to be addressed experimentally. The IFE program will benefit from continuing its emphasis on highrep-rate target production and target placement in the chamber, including near-term fabrication of materials and targets for testing of physics codes and the development of long-term solutions 
for mass production. The planned elimination of OFES funding for target fabrication is not consistent with its importance to the HI-IFE program.

\subsection{Drivers}

\subsubsection{Heavy-Ion (HI) Accelerator Drivers}

Worldwide experience with high-energy accelerators has long supported the prospect that an HIaccelerator driver for IFE can achieve the necessary efficiency, pulse-rate, and durability. As a result, the DOE has supported HI-fusion research since the late 1970s. Recent power-plant studies and research in the US and Germany predict additional favorable HI-fusion characteristics including efficient ion-target coupling, compatibility with indirect-drive targets and thick-liquidwall chambers, and the durability of focusing magnets.

The major near-term challenge for HI-fusion is to achieve the beam brightness on target required for ignition and high gain, as described earlier in Section 3.1.1. In the longer term, an experimental target-matter interactions program will be needed that is comparable to those presently underway for lasers and z-pinches. The near-term goal of the HI program, in the area of beam physics, is to determine the physics limits to intense HI-beam generation, acceleration, transport, pulse-length compression, and focusing that most impact the utility of HI beams to study high-energy-density physics (HEDP) and to drive IFE targets in the future.

This goal has been pursued in recent years by science campaigns (experiments, diagnostic development, theory, and simulations) primarily in the areas of:

1. High-brightness beam transport, to determine the technical requirements for preserving high brightness during transport of intense high-current ion beams,

2. Transverse focusing to millimeter spots, to develop a basic understanding of how beam-plasma interactions can be used to optimize the focusing of intense ion beams, and

3. Extensive advanced theory and particle simulations, to model the physics in the experiments, and to explore brightness degradation due to several non-ideal effects.

Research accomplishments in these areas are relevant not only to accelerators for HI fusion and HI-driven HEDP, but also to high-energy particle accelerators, non-neutral plasma physics, the physics of high-intensity particle beams, and particle-in-cell computer simulation development. (See Section 5)

Highlights of Recent Progress. [See ref 8 for details and references.] At low (mA-scale) beam currents, where beam-wall interactions are minimal, normalized beam emittance and brightness were preserved in transport through 86 accelerator elements, consistent with computer simulation predictions. Also at low current and low energy, ballistic focusing of a beam with $1 / 10$ of the charge density of a reactor scale beam was demonstrated experimentally, and neutralizing electrons from a hot filament reduced the focal spot size by an amount consistent with computer simulations. Furthermore, the measured brightness of high-current-density beamlets from an RFgenerated Argon plasma source exceeds the IFE brightness requirements.

In transport experiments with over 100 -mA beams, the initial low beam emittance $(0.5 \pi \mathrm{mm}$-mr with envelope parameters within required tolerances) exhibited negligible growth over 10 accelerator units, again as computer simulations predicted. Secondary electron production due to grazing-incidence HI impact of accelerator walls was found to be consistent with other 
accelerator data in preliminary experiments. Observations of large $(1-2 \mathrm{~cm})$ focal spots in vacuum, due to the effect of high space charge, becoming ten times smaller $(1.4 \mathrm{~mm})$ with preformed neutralizing plasma added to the transport chamber are in quantitative agreement with computer simulations.

Primary Remaining Issues and Research Plans. Experiments over the next three years (FY04-06, assuming the FY2004 budget and no growth) will use the existing 0.4-to-2-MV beam injectors, together with computer simulations, to address HI-beam losses induced by gas desorption and secondary electrons under space-charge-dominated conditions (space charge potentials $>1 \mathrm{keV}$ ). The ultimate goal of this work is to develop a predictive capability for beam loss scaling with magnet-aperture fill factor, field errors, and wall conditions. This research will be of broad interest to the accelerator community. Experiments supported by simulations will also address how the parallel ion temperature increases during strong longitudinal bunch compression under both un-neutralized, and beam-plasma-neutralized conditions. The role of instabilities due to transverse-longitudinal temperature anisotropy, and beam-plasma two-stream instabilities will also be investigated.

Over the next five years (FY04-08), 0.4-to-2-MV experiments and simulations will be carried out to give a predictive capability for focal-spot-size growth due to emittance growth from residual electric fields in target chamber plasma, from longitudinal beam temperature, and due to aberrations in the final focusing magnets. Over that same time frame, beam experiments at 2 to 5 $\mathrm{MeV}$, together with modeling, will evaluate the uniformity with which isochoric heating can be achieved in thin targets. These tests will use short-duration, tailored-current-profile HI pulses to optimize energy deposition for both HEDP and IFE applications. Over the next ten years, experiments intended to fully test integrated beam physics models will be needed. Ultimately, multiple-beam experiments at energies up to $200 \mathrm{MeV}$ will test the characteristics (pulse shaping, focusing, target shimming, etc.) predicted to be optimum for HI-IFE by integrated computer simulations together with hohlraum experiments on Z, NIF and possibly OMEGA. Relevant experiments on these facilities are possible today or in the near future.

\subsubsection{Krypton-Fluoride (KrF) Laser Drivers}

The decision to pursue $\mathrm{KrF}$ lasers as a fusion-energy driver was based on several attributes born out by experience with the NRL Nike facility. (Nike has been routinely used for laser target experiments in support of the NNSA mission since its completion in 1996.) KrF lasers have the short wavelength and demonstrated high beam uniformity for optimum laser-target physics, the brightness to achieve the required intensity on target, the ability to readily adjust spot size to follow the imploding capsule, a modular architecture for lower development costs, and a pulsedpower-based industrial technology that scales to an IFE-power-plant-sized system.

$\mathrm{KrF}$ lasers for IFE applications are pumped by counter injecting two electron beams into a laser cell filled with a mixture of krypton, fluorine, and argon. The laser-gas pressure (1 to $2 \mathrm{~atm}$ ) and the electron-beam parameters $(500-700 \mathrm{keV}, 100-500 \mathrm{kA}$, and $200-400 \mathrm{~ns})$ are adjusted to achieve uniform energy deposition across the cell, consistent with efficient laser operation. The laser gas, at around one atmosphere, is isolated from the electron-beam diode, immersed in vacuum, by a foil, which is supported by a structure known as a "hibachi". The laser beam propagates perpendicular to the electron beam direction. A recirculator flows the laser gas along the third axis to cool and quiet the lasing medium.

For the IFE application, the laser system must meet the fusion energy requirements for repetition rate $(5 \mathrm{~Hz})$, efficiency $(>7 \%)$, durability $\left(>3 \times 10^{8}\right.$ shots, or two years at $\left.5 \mathrm{~Hz}\right)$ and cost $(<\$ 400$ 
per Joule). These numbers come from power plant studies, and assume the target gain is at least 120. (Current target designs have gains at 150 or more.) The goal of the NRL Electra Laser is to develop the science and technology required to meet these requirements. Electra was started in 1999 and is funded by NNSA as part of the Congressionally mandated HAPL program. In addition, the HAPL program is developing final optics that are robust at high laser power and can survive target radiation and debris for the long lifetimes needed for IFE.

Highlights of Recent Progress. The Electra laser produces over $650 \mathrm{~J}$ of laser light in short repetitive bursts and uses technologies that can scale to a full-size system [9]. The requirements for repetition rate and the cost of the pulsed power component of the laser $(<\$ 10 /$ Joule) have been met, and overall efficiencies of about $7.4 \%$ have been projected based on experiments and modeling of the individual components. The ability to project such a favorable efficiency, which meets the requirement, is based on four advances: 1) Identification and stabilization of the "transit time instability" that previously plagued large-area electron-beam diodes. This was accomplished through experiments, theory, and 3D particle-in-cell computer simulations. 2) Development of an advanced diode/hibachi geometry that configures the electron beam to miss the hibachi ribs. These two advances increased the electron deposition efficiency into the laser gas from $35-40 \%$ to over $75 \%$. More than $80 \%$ is predicted for a fusion scale system. 3) Operation as a $\mathrm{KrF}$ oscillator, which leads to a projected intrinsic efficiency for an amplifier of greater than $12 \%$. 4) Development of an all-new, solid-state laser-triggered switch, which will be the basis for an ultra fast, efficient pulsed-power system [10]. This pulsed-power system is all solid-state, and therefore is expected to meet the IFE cost and durability requirements.

Primary Remaining Issues and Research Plans. The main outstanding challenge is to develop long-lived hibachi foils. A less challenging, but important issue is the development of long-lived amplifier windows. For the next two years the research campaign will concentrate on these two durability issues and a better understanding of the KrF physics. Foil development requires a coordinated research and development effort in materials, cathode physics, electron-beam transport, and thermal management. Preliminary experiments show the foil can be cooled by periodically deflecting the laser gas, but this needs further work. Window development requires high transmission fluorine resistant coatings that can survive the hostile environment of the cell. One promising candidate is already undergoing initial tests. KrF physics understanding is being carried out with a new KrF Physics Code called "Orestes" [11]. Orestes includes electron deposition, plasma chemistry, laser transport and amplified spontaneous emission (ASE). Deposition and laser transport are treated in 1D, whereas the ASE is modeled in 3D. The code follows over 22 species in at least 130 reactions. The code accurately predicts the behavior of several different $\mathrm{KrF}$ systems over a wide range of conditions, but needs further validation before it can be used to design the next generation system, which can cost $\$ 100 \mathrm{M}$ or more. This validation will be accomplished with experiments on the two amplifiers in Nike and Electra.

The next step, which is intended to start in 2-3 years, is to build a full-scale $(50 \mathrm{~kJ})$ beam line. The beam line will consist of eight identical e-beam systems, each about ten times the size of Electra. A fusion power plant is envisioned to have about 50-60 of these beam lines. The beam line will allow resolution of the science and technology issues that need a full-sized system to be properly addressed. This step is predicated on success with Electra, as well as commensurate advances in the other components needed for Laser IFE, including target physics, target fabrication, target injection, final optics, and chamber development. 


\subsubsection{Diode-Pumped Solid-State Laser (DPSSL) Driver}

Solid-state lasers have many applications in science, national security, and manufacturing. For IFE, however, several performance demands are unique, and require a directed R\&D program to achieve the goals for fusion as outlined above in the KrF section. The Mercury Laser research and development program, part of the HAPL Program funded by the NNSA, is intended to carry out proof-of-principal steps along the development path to meet these goals. Much of the science and technology foundation for the Mercury system is based on advances made in the ICF program in the early 1990's.

Highlights of Recent Progress. The Mercury laser program [7] has activated and operated one of two amplifier heads. In operation at the fundamental wavelength of $1053 \mathrm{~nm}$, Mercury has achieved $34 \mathrm{~J}$ in a $15 \mathrm{~ns}$ single-shot, and $23 \mathrm{~J}$ at $5 \mathrm{~Hz}$ for $10^{4}$ shots. The efficiency was $4 \%$ in a 5-times diffraction-limited beam. In order to reach this point, it was necessary to develop special diode arrays, an inexpensive mass-producible silicon heatsink (which subsequently became the subject of a technology-transfer action), a new bandwidth-enhanced front-end for the laser (developed with LLE University of Rochester), and the first thermally compensated high-power Pockels cell.

The Mercury Laser DPSSL Program achievements are characterized by scientific investigation followed with technology demonstration. For example, the decision to use the Yb:S-FAP gain medium required an understanding of the $\mathrm{Sr}_{5}\left(\mathrm{PO}_{4}\right)_{3} \mathrm{~F}$ (S-FAP) materials science issues including the chemical compositions and lattice structures that characterize transitions in the phase diagram. The compositions and structures of defects led to an appreciation of the instabilities that exist at the melt-solid interface during crystal growth. Eventually, laser medium crystals exceeding requirements were grown using an off-stoichiometry melt, reduced $\mathrm{YbF}_{3}$ dopant, and highly conditioned power to stabilize the melt-solid interface.

The development of a scalable optical architecture of the system is another highlight of the research. Detailed models were developed by accounting for the quasi-three-level character of the $\mathrm{Yb}^{3+}$ laser ion, pump bleaching, and gain; by linking to ray-trace models of the diode pump light delivery via a hollow lens duct; and by coupling a nonlinear model of laser beam propagation. This work led to the development of a new laser architecture that is more robust against nonlinear ripple growth and damage than previous systems.

Another notable multidisciplinary achievement was the development of the helium gas-cooled amplifier. Aerodynamics, heat removal, and stress-optic calculations were combined to design the first high-speed (Mach 0.1) gas-cooled laser slabs. This achievement eventually led to the successful demonstration of the 7-slab (34-J) amplifier.

Primary Remaining Issues and Research Plans. The goals for the Mercury Laser are $10 \mathrm{~Hz}, 100 \mathrm{~J}$ 3 -ns pulse, and $10 \%$ efficiency. The efficiency goal is for unconverted $(1053 \mathrm{~nm})$ unsmoothed light. When the frequency is tripled, the beams are smoothed for IFE, and other practical matters are taken into account, the efficiency is predicted to be around 7\%. Significant research and development effort will be devoted in the near-term to implementing the front-end operation with spectral - temporal - spatial tailoring; activation of the second amplifier head with 7 more laser slabs, improved damage resistance for several optics in the system (including the final optics research described below), and implementation of a faster and more extensive control system to rapidly detect optical damage and diode-tile failure. The major scientific issue that demands fundamental understanding is that of optical damage, including the field enhancements arising from surface cracks of different morphologies. It is also important to note that much of the 
Mercury research is directly related to the development of high-average-power short-pulse lasers needed for the Fast Ignition concept.

After the 100-J system is functioning at the fundamental frequency, the engineering science effort will focus on designing and implementing a high-average-power frequency-conversion module to yield 351-nm light.

The successor to the Mercury Laser is envisioned as a 4-kJ aperture system. A pressing issue that must be addressed is the management of amplified spontaneous emission (ASE) and stimulated Raman scattering (SRS), which must be modeled in great detail and then experimentally verified at the appropriate scale. Furthermore, larger laser slabs $\left(20 \times 30 \mathrm{~cm}^{2}\right.$ compared to $4 \times 6 \mathrm{~cm}^{2}$ currently) must be fabricated. Several avenues are being investigated including "flat interface growth" to alter the convection currents in the melt, and water-glue bonding which entails identifying a chemically compatible adhesive. Cost is an issue, so a commercial source of lowcost diode arrays must be established.

Final Optics. A HAPL program on final optics for both KrF and DPSSL lasers has begun testing a design concept based on grazing incidence metal mirrors. The radiation and laser-induced threats have been characterized, and facilities for sub-scale components have been assembled to measure laser, $\mathrm{x}$-ray and ion damage. The mirrors have a reflective coating (such as aluminum) bonded to a neutron-damage-resistant substrate (such as silicon carbide). The research has shown that this type of mirror has high reflectivity ( $>99 \%$ has been measured), and high durability ( $>80 \%$ above anticipated laser fluence at prototypical wavelengths and for $10^{5}$ shots). However, the durability must be confirmed in large-scale samples, and eventually, with shot lifetimes required for IFE. The material microstructure and bonding of the reflective coating has been studied in order to improve damage resistance. The damage threat has been modeled for ions and $\mathrm{x}$-rays. Initial x-ray testing has demonstrated single-shot survival and higher shot counts are now being obtained.

\subsubsection{Z-Pinch Drivers}

A Z-Pinch IFE program was initiated in 1999 following the successes on the Z facility at Sandia, which demonstrated outstanding overall efficiency (15\% of the stored electrical energy converted into x-rays). One purpose of the program was to investigate ways to increase the repetition rate of this x-ray source for indirect-drive IFE. The concept of a recyclable transmission line (RTL) was developed to address the principal issue of physically connecting a repetitive pulsed-power driver to a fusion target. In contrast to the $\mathrm{Z}$ facility where a massive $(8000 \mathrm{~kg})$ transmission line must be removed and cleaned between experiments, in the RTL concept the transmission line is designed to be a low-mass $(50 \mathrm{~kg})$ structure that is destroyed along with the target on each shot. By making the RTL from the chamber coolant or from a material that is easily separable from the coolant materials, the RTL materials can be continually recycled to manufacture new RTLs. The continuous on-site manufacturing of the RTLs with the recycled materials means that only a small inventory of transmission lines would be needed. With relatively high per-pulse yields of about 3 GJ, the rep-rate per chamber can be relatively slow (about $0.1 \mathrm{~Hz}$ ), for a 1-GW(e) power plant. Such a plant would have 8 to 10 chambers with a common RTL and target factory. The RTL concept eliminates the problems of final optics, high-speed target injection, and accurately pointing many beams at a fast moving target. Since it is a relatively new approach, the Z-Pinch IFE program is in the concept development phase of determining if it is feasible and potentially economically attractive for energy production. 
The primary research activities have been the following:

1. RTL science, including initial electron flow, power flow uniformity, minimum mass, electrical conductivity, and structural properties, etc., has been investigated in order to determine the basic feasibility of the RTL concept;

2. Repetitive pulsed power concepts have been studied to determine the most appropriate repetitive pulsed-power technology for energy;

3. High-yield target studies have been carried out for yields of $\sim 3 \mathrm{GJ}$; and

4. Conceptual Z-Pinch IFE power plant studies have investigated thick liquid-wall chambers, RTL/target manufacturing feasibility and cost, RTL operational cycle with vacuum and electrical connections, activation analysis, waste stream analysis, etc.

It is noteworthy that in the last two years, ICF implosion experiments using the $\mathrm{Z}$ machine radiation drive have coupled nearly $40 \mathrm{~kJ}$ of $\mathrm{x}$-ray energy to a capsule and achieved the first fusion neutrons $\left(\sim 8 \times 10^{10} \mathrm{D}\right.$-D neutrons, an order of magnitude higher than has been produced from any other inertial fusion facility) from a $z$-pinch driven capsule implosion. These experiments have been well modeled, giving further impetus to the Z-Pinch IFE program.

These initial IFE studies were supported by internal (LDRD) funds at SNL. In FY04, a larger program within NNSA is being started, supported by a Congressional initiative of $\$ 4 \mathrm{M}$.

Highlights of Recent Progress. [See refs 12 and 13 for details and references.] Transmission-line electrical-power-flow-uniformity experiments carried out at the 10-MA level on Saturn showed that the electron flow in RTL candidate material coatings of tin, Al, and stainless steel all initiated rapidly when subjected to high electric gradients such as those that would occur in an RTL in a power plant. RTL masses of $50 \mathrm{~kg}$ would have very low electrical losses $(\sim 10 \%)$. Structural analysis shows that a full-scale RTL of mass $50 \mathrm{~kg}$ would survive (without buckling) in a chamber background pressure of 10-20 Torr. Manufacturing studies show a ferritic steel RTL cost of about $\$ 3.60$ each (including capital costs) at the $90 \%$ confidence level (which is consistent with the allowed RTL budget assuming 3 GJ yields).

Target studies for 3-GJ yields from the two leading Z-Pinch target-driver configurations (doubleended z-pinch and dynamic hohlraum) require 30-36 MJ of x-ray drive (gains of 80-100), so that both target concepts are contenders for Z-Pinch IFE.

An initial (not optimized) power-plant study was completed for a 1-GW(e) Z-Pinch power plant. The study included a low-activation ferritic steel RTL, the complete recycle operation for these RTLs, vacuum and electrical connections, thick liquid (Flibe) walls, and automation of the RTL process.

Primary Remaining Issues and Research Plans. At this early stage in development, the key issue is still feasibility of the RTL concept. Questions of RTL shape, inductance, material (frozen coolant or easily separable material), mass, electrical characteristics, structural characteristics, manufacturing, recycling, activation, and cost must still be addressed. The primary pulsed-power science issue is the physics of power flow in a high-power-density transmission line. The powerflow limits and the optimal RTL configuration for power flow (coax, triax, convolute, etc.) must be explored. Chamber interface issues, including electrical/vacuum connections, sensitivity to post-shot electromagnetic-pulse/plasma/debris flow up the RTL, and shielding of sensitive power flow feed parts must be examined. Proof-of-principle-scale RTLs for the 1-MA level need to be designed, built and tested. Design, cost, and scheduling of an RTL demonstration on Z needs to be done. RTLs would be of benefit to $\mathrm{Z}$ and NNSA by reducing the time required between shots 
on the facility. A baseline 60-MA RTL design will be developed to serve as a focus for the continuing development studies.

Possible approaches for the pulsed power to drive the RTLs must be assessed, including Marx generator/water line technology, magnetic switching technology, and LTD (Linear Transformer Driver) technology. A study of long-lifetime repetitive switches will also be necessary. Plans also include the design, construction, and testing of a 1-MA, 1-MV, 100-ns, $0.1-\mathrm{Hz}$ repetitive driver as a proof-of-principle-scale driver.

Since a 3-GJ yield is larger than typical yields for other IFE concepts, shock mitigation with thick liquid wall coolant streams is an issue that needs to be studied. Scaled shock experiments with explosives and water (or other liquid) flows can be used to address this issue. Codes that are benchmarked against these experiments can then be used to model full-scale reactors.

Longer term issues are largely related to optimization, including the optimum high-yield target for the double-ended z-pinch and dynamic-hohlraum configurations and the necessary powerflow geometry for each, the optimum repetition rate, and the development of a practical RTL insertion process at $\sim 0.1-\mathrm{Hz}$ rep-rate. All of the parts must, of course, fit together in a viable power-plant configuration, including a survivable chamber, RTL/target production, robotic operation, recycling of materials, heat cycle optimization, etc. Therefore, a complete proof-ofprinciple system to demonstrate RTL/z-pinch insertion, vacuum/electrical connections to the primary pulsed-power system, firing of the z-pinch, and removal of the remnants, must be designed, built and tested at $\sim 0.1 \mathrm{~Hz}$ as a precursor to design and construction of larger test facilities.

Comment on Drivers: The OFES-funded HI program concentrates on the scientific understanding needed to accelerate and focus the HI beams needed for IFE target compression. The NNSA-funded KrF and DPSSL HAPL program leverages heavily on the NNSA scientific program for laser-driven ICF, and emphasizes the technical development of efficient, durable, high-rep-rate laser drivers for IFE application. The NNSA-funded Z-Pinch-IFE program leverages off the NNSA-funded Z-Pinch ICF program and has concentrated on concept development of technology permitting a $0.1-\mathrm{Hz}$ repetition rate with the recyclable transmission lines needed for IFE application.

Assessment Analysis: In assessing the quality and appropriateness of the work done on the HI and HAPL drivers, the Panel made primary use of the research goals to be achieved in 5 years, as set down by the community and endorsed by FESAC [4]. The Panel was particularly impressed with the following examples of progress. For the HI program: the key science objectives were (i) to carry out validating single-beam, high-current experiments at 10-times-higher line-charge density and, (ii) to perform focusing and chamber transport experiments at intermediate scale. The HI program has met these objectives, with successful experiments carried out at the 100-mA beam level and very successful demonstration of plasma neutralization assisted focusing. For the laser drivers: the key technical and scientific objectives were to achieve (i) an energy of several hundred joules in a laser architecture scalable to $2 \mathrm{MJ}$ at a cost of $\leq \$ 500 / \mathrm{J}$; (ii) wall-plug efficiency of $6-10 \%$ at a repetition rate of $5 \mathrm{~Hz}$; (iii) reliability of $10^{5}$ to $10^{8}$ shots between maintenance cycles; and (iv) irradiation uniformity of $\leq 0.3 \%$. The HAPL KrF program has made very good progress in reaching most of these goals. The following have been achieved but not yet simultaneously: Energy greater than $650 \mathrm{~J} /$ pulse at repetition rates of $1 \mathrm{~Hz}$ and $5 \mathrm{~Hz}$, beam uniformity of $0.2-0.3 \%$ single shot, and efficiency greater than $7 \%$ (predicted from advances in the principal individual components). Only the reliability (owing to the hibachi foil lifetime) 
remains significantly below (factor of 100) the 1999 objectives level. The HAPL Mercury Laser DPSSL program has also made very good progress by achieving simultaneously $4 \%$ efficiency (at the $1053 \mathrm{~nm}$ fundamental) at 23 Joules and $5 \mathrm{~Hz}$ for $10^{4}$ shots. Producing the necessary beam uniformity at $100 \mathrm{~J}$ and $7 \%$ efficiency (at $351 \mathrm{~nm}$ ) on target have yet to be achieved.

Since the outstanding progress in z-pinch x-ray generation was just beginning to be reported in 1999, there was no comparable set of goals established by FESAC for the Z-Pinch driver. Therefore, the Panel based its assessment of quality and appropriateness of research on the achievement of $\mathrm{x}$-ray power and energy levels from the $\mathrm{Z}$ machine to the level needed to carry out ICF compression experiments, the extensive list of recent conference and peer reviewed publications, and the substantial progress reported to us on the concept development of the recyclable transmission line necessary for IFE application of the Z-Pinch driver.

Finding: The three main approaches (HI accelerators, HAPL, and Z-pinch) are at different levels of maturity. The balance between the science and technology emphasis necessarily varies. The recent progress related to these approaches is substantial and the quality of the science and engineering research is excellent. All approaches are currently on track for developing the science and technology to properly evaluate their potential for IFE.

\subsection{Fast Ignition}

The Fast Ignition (FI) concept employs two drivers -- one for compression, which can in principle be any of the four types described in Section 3.2 ( $\mathrm{KrF}$ and solid state lasers, HI accelerators, and Z-Pinches), and one to ignite a portion of the compressed fuel. The igniter laser must meet challenging requirements including high brightness $\left(10^{19}-10^{20} \mathrm{~W} / \mathrm{cm}^{2}\right)$ at high energy $(\sim 10-100$ $\mathrm{kJ})$, a short pulse $(\sim 10-20 \mathrm{ps})$, and small spot size $(\sim 20-40 \mu \mathrm{m})$. Solid-state petawatt lasers that employ the Chirped Pulse Amplification (CPA) technique are today the most advanced drivers for FI but a $\mathrm{KrF}$ laser using transient Raman scattering is also viable.

Fast Ignition potentially offers several attractive features: higher gain, lower driver energy, and more compression-driver flexibility. Without a hot spot, more fuel can be efficiently compressed to the required areal density, $\rho \mathrm{R}$, where $\rho$ is the fuel density and $\mathrm{R}$ is the fuel radius. Most assessments of the gains achievable with FI assume that the fuel has been compressed to a uniform-density sphere. The main requirement -- and challenge -- for FI is to deliver the ignition energy to the compressed fuel. Petawatt laser energy is nominally deposited in the coronal plasma surrounding the compressed fuel at (or below) the critical plasma density, which is $\sim 10^{-5}$ of the compressed fuel density $\rho$. The igniter-laser-beam energy deposition results in a relativistic electron beam. Ignition depends on the successful propagation of that electron beam to the fuel and the effective heating of a small portion of that fuel.

The most developed FI scheme involves the so-called "cone focus" target shown in Figure 1g. In this concept a cone is inserted into a fuel capsule with its tip positioned near where the fuel layer will be at the end of the compression. The cone is made of dense high- $\mathrm{Z}$ material to minimize wall motion during the $\sim 10 \mathrm{~ns}$ fuel compression. At present both indirect and direct-drive approaches utilize this cone design, although the more limited intervening plasmas associated with direct drive may allow other coronal "plasma clearing " techniques such as laser channeling. The cone may provide additional benefits by focusing the igniter beam energy, as indicated in recent experiments in Japan $[14,15]$. 
Highlights of Recent Progress. Numerous experiments have shown energy-conversion efficiencies of $\sim 40 \%$ from a petawatt-laser beam into relativistic electrons at relevant plasma conditions for FI. Transport experiments have highlighted the importance of background plasma conditions as well as identifying and exploiting high-energy ions. Fuel assembly experiments done primarily in the US with cone and hemispherical targets utilizing both indirect and direct drive have achieved core densities of $\sim 20-30 \mathrm{~g} / \mathrm{cm}^{3}$ and are well modeled by hydro codes.

The most impressive FI experiments have been performed at the GEKKO facility in Japan (see Appendix K). With a 9-beam cone-focus direct-drive implosion using $\sim 2.4 \mathrm{~kJ}$ of $532 \mathrm{~nm}$ light and a one-beam "igniter" laser with $\sim 300$ joules at $1053 \mathrm{~nm}$ in a $\sim 0.5$-ps pulse, the experiments showed a three-order-of-magnitude increase in neutron yield (to $\sim 10^{7}$ ) compared to the $2.4-\mathrm{kJ}$ implosion alone (at $\sim 10^{4}$ ). A self-consistent increase in ion temperature from $150 \mathrm{eV}$ to $800-1000$ $\mathrm{eV}$ was also observed. These increases occurred only when the igniter pulse was timed to arrive at the time of peak compression. Although conditions achieved in these experiments are far removed from the regime in which ignition and gain would occur, their success has generated optimism for the prospects for FI.

Primary Remaining Issues and Research Plans. Efficient transport of the relativistic electrons through the plasma corona surrounding the imploded shell and into the high-density fuel is the key scientific issue of the FI concept, and it is a topic of much current research. The giga-amp energetic-electron currents must be largely neutralized by a return current that is composed of relatively cold, highly collisional electrons in order for the relativistic-electron energy to be efficiently transported through the coronal plasma and into the dense core. A wide range of plasma collective phenomena is predicted to occur that could inhibit the energy flow into the core. Experiments and theoretical analyses have shown that the background plasma conditions have a major impact on the transport properties of the high-energy electrons. FI experiments that study energy transport must therefore involve plasmas that mimic the conditions found in an imploded DT target. This represents a significant experimental challenge. There also are substantial computational challenges; these include relativistic laser-plasma coupling and selfconsistent, high-energy electron transport with neutralization by cold, collisional electrons, all in three spatial dimensions. New computational methods are being developed to tackle these problems.

The relative ease of transport of energetic ions through the low-density plasma surrounding dense fuel, plus the ability to focus these ions have recently motivated FI concepts that employ ions. Limited experiments to date have shown maximum efficiencies of converting the laser energy to energetic ions of only $\sim 10 \%$, but laser-produced ions have already been used for isochoric heating of plasmas to temperatures of order 60-70 eV [16,17]. Much of the research in the generation and applications of petawatt laser produced ions has been funded by an OFES innovative concepts initiative. This new research topic also has attracted significant interest for its other possible applications, such as radiography, compact accelerators, and novel ion sources.

While there has been substantial progress in FI target design, there is as yet no self-consistent 2D implosion for a cone-focus target that achieves the ideal final state of a uniform density sphere. The limited number of experiments that do not use a cone have not been encouraging.

Research over the next several years will need to focus on the issues of fuel assembly and energy transport at relevant conditions. Modeling of both direct and indirect drive implosions that achieve nearly uniform density core conditions -- validated by experiments on facilities such as OMEGA, Z, NIF, and GEKKO -- is critical. For instance, hemispherical capsule implosions have already been radiographed on $\mathrm{Z}$, in preparation for future FI physics experiments. Integrated 
experiments that, if successful, would be "proof of principle" of the FI concept, should soon be possible. If such experiments show great promise, up to 20 beams of NIF could be configured for short pulse operation for a demonstration of Fast Ignition.

Assessment Analysis: FI is an exploratory concept that was funded (at a low level) by the IFE program subsequent to the FESAC report [4] where key 5-year goals for the IFE program were established. As noted above, the promise of FI lies in higher gain and/or lower driver energy for IFE, so quality can be assessed by noting progress in establishing the physics basis to realize that potential. Of particular note was the recent demonstration of increased neutron yield achieved with a 300 Joule FI laser pulse onto a 2.4-kJ 9-beam implosion target plasma in the GEKKO facility. In addition, experiments have shown $40 \%$ conversion of petawatt-laser energy into relativistic-electron-beam energy. Well-diagnosed cone-implosion experiments achieved 20 to 30 $\mathrm{g} / \mathrm{cm}^{3}$ compressed core densities.

Finding: Each of the approaches to IFE may benefit if the technique of Fast Ignition proves effective. Recent experiments on the GEKKO laser in Japan have offered very encouraging indications that efficient igniter-beam energy transport to and heating of a compressed core are being observed. However, since FI is at an early stage of development it would be premature for any of the IFE approaches to rely on the success of FI to achieve an attractive fusion energy system. During the next several years, there is an opportunity to assess the potential of the FI concept utilizing facilities in both Japan and the US (OMEGA, Z, and possibly NIF) through modest OFES investments.

\subsection{Chambers and Integrated Concept Studies}

\subsubsection{Chamber Science and Engineering}

IFE targets emit neutrons, ions, x-rays and gamma rays. Following each inertial fusion target explosion, particles and radiation propagate into the surrounding medium, interact with materials in various states of matter, and finally are converted into heat and, in the case of breeding blankets, fuel to supply further fusion reactions. Depending on the chamber design, the target emissions may interact with solids, liquids, gases and/or plasmas. Chamber science and engineering research in the US explores these basic interactions and attempts to solve key questions related to chamber operability and survival. This research has been carried out with funding from NNSA and OFES.

Highlights of Recent Progress. The HAPL program has adopted a reference chamber concept based on solid first walls, e.g., tungsten armor on steel [7]. The choice was based on the inherent simplicity of the approach, and the fact that a solid wall is feasible with a direct-drive target whose emissions are dominated by energetic ions and neutrons, and not prompt x-rays. (Only $\sim 2 \%$ of the target output is in the form of high-energy x-rays.) Progress has been made on the detailed characterization of post-burn target emissions, energy transport through a gas background and absorption in the surrounding armor. Materials studies have been carried out to better understand the prompt effects of pulsed ion deposition in first-wall armors, including thermomechanical effects as well as ion implantation and transport. Evolution of the chamber environment following target explosions has been simulated numerically in order to establish the ability to propagate targets and driver beams with a repetition rate consistent with economic energy production. 
OFES-funded chamber research is currently focused on a chamber concept based on neutronically thick liquid jets to handle the indirect-drive target emissions. This chamber is consistent with the needs of HI fusion and has the potential to simplify the fusion chamber material development challenges. Progress has been made in a range of technical disciplines, including thermalhydraulics of high Reynolds number jets, aerosol creation and transport, liquid response to highenergy-density $\mathrm{x}$-ray bursts and isochoric neutron heating, and transport of target debris into background gases and magnetic fields. A small fraction of the program has explored laser-IFE issues such as mirror damage and laser propagation.

SNL internal funds have been used for initial studies of Z-Pinch chambers, including neutronics studies, activation analysis, and initial analysis of the RTL breakup following the capsule explosion.

Primary Remaining Issues and Research Plans. The remaining key issues and R\&D needs for dry-wall and thick-liquid chambers are quite different. For dry-wall chambers, long-term survival of the chamber armor remains the most visible feasibility issue. The HAPL program has undertaken a series of experiments and modeling to understand materials responses at a microscopic level and to develop damage-resistant armors using engineered microstructures. The experiments are performed on irradiation facilities ( $\mathrm{x}$-ray, ion, and laser) at several national labs and universities. "Chamber clearing" (how the chamber returns to a state that allows insertion of a new target and delivery of the driver energy to the target) is an important issue for all IFE approaches. For solid-wall chambers, the HAPL program has developed a code that uses compressible Navier-Stokes equations and an adaptive-mesh grid to model the post-shot behavior in the chamber [7]. Predicting these dynamic interactions requires a detailed understanding of hydrodynamics and radiation transport in the partially ionized afterglow plasma. The modeling will continue, and experiments to test this modeling will use a recently completed small rep-rated laser facility at UCSD.

For thick-liquid chambers needed for HI fusion, the dominant near-term issue is establishing the basic flow configuration while meeting the requirements for neutron shielding and HI-beam transport. Studying the formation and hydrodynamics of stationary and oscillating jets has been the focus of the OFES chamber program over the past 5 years. The results on shape control and droplet formation are encouraging, and warrant a more integrated examination of the complete flow geometry. The generation and transport of aerosols requires further study in order to demonstrate successful propagation of targets and HI beams into the liquid pockets. Rapid absorption of $\mathrm{x}$-rays in liquid surfaces can lead to intense non-equilibrium responses, such as spinodal phase decomposition, with absorbed energy density as high as $10^{12} \mathrm{~J} / \mathrm{m}^{3}$, well into the HEDP regime. Future progress on these issues is in jeopardy due to the planned closeout of IFE chamber research funded by OFES.

Thick-liquid chambers are also under study for Z-Pinch IFE. The key near-term issue is to establish the basic flow configuration that will provide adequate shock mitigation to the structural wall, given the higher fusion yield ( $3 \mathrm{GJ}$ ) per target being planned for this approach.

Assessment Analysis: Much progress has occurred during the past five years in chamber research. A large number of refereed journal publications and presentations at international conferences have occurred during this period. This impressive list speaks clearly to the issue of depth and quality of the research being carried out in this difficult area, which is at the intersection of several traditional disciplines. The Panel notes that all of the elements of a thick liquid wall chamber have been explored both empirically and numerically. Flow geometries include stationary circular and sheet jets, oscillating sheet jets and vortex flows. The flow 
formation and hydrodynamics have been demonstrated, including extensive research on flow conditioning that is needed to maintain laminar conditions and avoid hydrodynamic sources of droplet ejection. Another topic of research that has progressed significantly is the understanding of the mechanisms for aerosol production due to pulsed energy deposition. These mechanisms include isochoric heating, phase explosion, and shock-induced liquid fracture.

Finding: The dynamic response of chambers following target explosions is a critical scientific issue in determining the repetition rate and durability and hence the ultimate attractiveness of all IFE concepts. Important chamber issues requiring further work for their resolution include thick liquid chamber dynamics and shock mitigation, aerosol generation and transport, armor survival, and chamber clearing. The closeout of chamber $R \& D$ being planned by OFES is not consistent with its importance to the HI-IFE program.

\subsubsection{Integrated Concept Studies}

Integrated concept studies play an essential role in the IFE program by providing self-consistent integrated design options based on the available database as well as technical requirements needed for a viable fusion reactor. The studies foster innovations that help move the science and technology in directions that will meet the ultimate needs. Through analysis and systems integration, the output of concept studies is used to direct R\&D towards the highest leverage and most critical tasks. New results from R\&D programs are then fed back into concept studies in a continuous cycle of improvement. These studies are needed to identify not just the most effective experiments that need to be conducted in the near term, but also the most cost-effective routes to the evolution of the experimental, scientific and technological programs.

Design requirements are derived from the demands of the marketplace, including reliable plant operation, tritium self-sufficiency, efficient energy conversion, minimal environmental impact, and economic viability. For example, the need for high thermal conversion efficiency drives the design process for both liquid and solid chambers. Examples of how this impacts R\&D include:

1. Flibe coolant was chosen for the HI and Z-Pinch liquid chamber largely because it gives reasonable energy efficiency, considering both pumping power and thermal constraints.

2. The operating temperature of dry-wall chamber armor determines damage mechanisms and influences direct-drive target survival during injection into the hot chamber.

Highlights of Recent Progress. A 3-year OFES-funded activity was recently concluded under the umbrella of the ARIES concept studies team. This research explored design windows for chambers, including key driver and target interfaces. Various combinations of target, driver and chamber were explored in order to establish design windows and to identify key research needs. This activity has been completed with no immediate plans for ongoing research.

Also within OFES, the Virtual National Laboratory for HI Fusion has recently published a "robust point design" based on a multi-beam induction LINAC driver [18]. This point design defines key technologies, nominal parameters and design requirements for a self-consistent energy source based on $\mathrm{HI}$ indirect-drive targets and neutronically thick liquid-wall chambers.

Initial R\&D needs for the High Average Power Laser program were defined largely based on earlier studies, such as the Sombrero KrF Concept [19], ARIES [20], a DPSSL concept study [21], and new target physics results [22]. The HAPL program has spent the last four years experimentally evaluating the assumptions and parameters that went into these studies. 
Primary Remaining Issues and Research Plans. New integrated concept studies become appropriate when substantial new research results become available. Both the HAPL and Z-Pinch programs are poised to embark on such studies. Based on results from the past 3 years of R\&D, the multi-institutional HAPL team will soon undertake a new concept study; the initial Z-Pinch power plant study (ZP-3) [12] will be refined and improved during the coming years as a result of new physics results.

Assessment Analysis: One of the most important measures of the quality of power plant studies is the impact that they have on the base program of R\&D. In the IFE program, as in the MFE program, there are many recent cases in which integrated concept studies have affected $R \& D$ programs. As a noteworthy example, requirements on cyrogenic target survival emerged from recent power plant studies. In these studies, trade-offs between the minimum gas pressure required to protect dry walls from ions and the maximum gas pressure allowed to avoid target degradation (and hence, unreliable implosion) were clearly articulated. These results significantly affected research in the areas of target physics, target design and engineering, and chamber armor.

Finding: Integrated power plant studies play an important role in identifying critical issues and in driving innovation in science, technology, and engineering for IFE. The planned elimination of integrated studies is not consistent with their importance to the OFES HI-IFE program. 
March 29, 2004

\section{KEY NEAR-TERM ISSUES}

\subsection{Summary of IFE Issues}

The primary issues currently facing the various elements of the IFE program were identified in the previous section. Now this section of the report summarizes and organizes these issues, and then selects from them the most critical near-term feasibility issues.

Some preliminary comments are necessary to place in proper context both the scientific issues, as requested in the charge letter, and the prevailing views of the IFE community concerning overall program directions. These comments provide the motivation for the way the summary material is organized.

The IFE program is strongly guided by its ultimate goal of fusion energy. The accomplishment of this mission will require research in numerous science and engineering disciplines, as well as integrated systems analysis. The science research covers a rich variety of physical phenomena including plasma physics (neutral and non-neutral plasmas), high-energy-density physics, materials science, nuclear physics, and strongly non-linear hydrodynamics. There is a similar richness in the basic engineering science and technology research required for target fabrication, driver development, and chamber design. The prevailing view in the IFE community is that the most efficient way to achieve its ultimate energy mission is for the required R\&D in science and engineering to be carried out in the context of an integrated system, always keeping the end product and its explicit requirements in mind. Consequently, although commercial fusion energy, either MFE or IFE, is still decades away, it is this long-term view that guides the choice of problems to be investigated in the near term -10 years or less. These near-term issues are the basis of the research plans described in the previous section.

In this context, the three main approaches to IFE - HI, HAPL, and Z-Pinches -- must address issues in the same three major component areas - targets, drivers, and chambers. Below is a brief, generic description of the overall issues that must be resolved for each of these components within the context of a fusion system that is cost competitive and environmentally attractive. Following this generic description is a more detailed discussion that focuses on the most critical near-term issues, directly addressing the request in the charge letter. These issues are organized approach-by-approach, consistent with the need for a long-range integrated plan.

- Target Issues: The overall goal is to produce a target design that has sufficient gain for fusion energy, and that can also meet the requirements for the rates of fabrication and injection into a hostile reaction-chamber environment. The near-term issues focus on hydrodynamic instabilities, and symmetric and asymmetric implosion dynamics needed for ignition and high gain. IFE target research has been primarily focused on symmetric implosions although the relatively new and novel idea of Fast Ignition requires an asymmetric implosion. The long-range issues involve the design, characterization, and cost-effective manufacture of targets, and their injection or placement in the chamber.

- Driver issues: The overall goal is to develop a driver that can meet the fusion energy requirements for power focusing, efficiency, repetition rate, and durability. These requirements are, of course linked to the performance of the target. For example, the lower the target gain, the higher the required driver efficiency. Thus, the near-term research issues are driver efficiency, power focusing, pulse shaping, overall target energy gain, and energy absorption by the capsule. The long-term issues are durability and reliability at high-rep-rate, 
especially of the energy-delivery or focusing components that are exposed to the chamber environment.

- Chamber Issues: The overall goal is to design a chamber that can meet the requirements for wall durability, while allowing highly reliable target injection/placement, and efficient driverenergy delivery to the target. The near-term issues focus on radiation transport, rapid energy deposition by neutrons, charged particles, and x-rays, on wall materials, shock stresses on the walls, and understanding the post-pulse chamber environment and, for HI and HAPL, its impact on beam focusing and propagation and on target injection. The related long-term issues involve the development of the survivability of first walls and high rep-rate capability, while allowing the accurate and rapid injection/placement of targets into the chamber. These capabilities must also be consistent with efficient energy conversion, tritium production, and environmental acceptability.

The near-term issues from the above list were examined to identify which ones appear to be the most critical. The critical issues of ignition and burn affect all of the approaches, but those challenges are being addressed mainly by the ICF program. For that reason, they are not considered here as candidates for the single most important near-term issue facing each IFE approach and the FI concept. The remainder of this section describes these issues and how progress therein can lead to substantial improvements in the long-range overall desirability of IFE.

\subsection{Heavy-Ion Fusion}

The key near-term challenge for HI fusion is the focusing of HI beams to a very small spot size $(\sim 2-5 \mathrm{~mm}$ radius) and compressing the pulse length to a few nanoseconds. This focusing and compression must be accomplished at progressively higher intensity levels to ultimately meet the requirements of a high-gain fusion target.

The solution to the problem requires substantial research in plasma physics. The electric space charge of a non-neutralized ion beam tends to spread the beam out. This effect can be mitigated by introducing plasma into the beam path. Smaller beam spot sizes are achieved, because the plasma neutralizes the space charge, but at the expense of some growth in the beam temperature. This temperature and any small spread in energies about the beam velocity will limit how small the spot size and pulse duration can be made.

The near-term goal is to develop a sufficient understanding to identify techniques that minimize the effects of space charge and temperature on beam spot size at the target. This requires that the beam dynamics be controlled with high precision along the entire beam trajectory through the accelerator and target chamber. A fundamental understanding of the collective processes and nonlinear dynamics of intense, high-brightness, HI beams, and accelerator systems is essential to progress.

The research plans for the next five years will make use of the existing 400-keV and 2-MeV ionbeam facilities and existing particle-in-cell codes in the HI program. The science campaigns (experiments and simulations) will address key intense beam physics issues in high-brightness beam transport, longitudinal bunch compression, and final focusing with plasma neutralization. With some enhancements, these facilities and codes will also be sufficient for achieving a successful outcome to the OFES/OMB 10-Year Measure for Inertial Fusion Energy (IFE) and High Energy Density Physics. 
March 29, 2004

The most critical near-term issue facing $\mathrm{HI}$ fusion can thus be summarized as:

Physics limits to the maximum phase-space density of space-charge-dominated HI beams and the resulting implications for HEDP and fusion ignition.

\subsection{Laser Fusion}

For laser fusion the critical near-term goal is to demonstrate a path to achieving an overall energy gain that is sufficient for practical fusion energy. There are two key elements for achieving this goal. One is to produce a reliable, durable laser that can meet the IFE requirements for efficiency. This is the motivation for the $\mathrm{KrF}$ and DPSSL research in the HAPL program. The second element involves the design of higher gain targets that are readily fabricated in large numbers. Much of this research is carried out under the auspices of the NNSA ICF program. [This ICF-funded activity is mentioned here because target gain is of particular importance to the HAPL approach. The efficiency projected for lasers is lower than for HI or Z-Pinch, and therefore higher target gains are required.] Note that these two challenges are inextricably linked, in that capsule gain can be traded for laser efficiency.

$\mathrm{KrF}$ lasers have very smooth beam profiles and a fundamental wavelength of $248 \mathrm{~nm}$ that is ideal for coupling to a direct drive target. The primary near-term challenge is to discover ways to maintain the predicted efficiency and realize durable long-lived "hibachi" foils through which electron beams are injected into the laser gas. This will require research into electron-beam generation, electron-beam physics, and the physics and chemistry of materials. Furthermore, these foils cannot be investigated in isolation but must be developed as part of a fully integrated laser system. Based on current $\mathrm{R} \& \mathrm{D}$, which show $\mathrm{KrF}$ laser efficiencies predicted to be $7 \%$ or greater, and target gain calculations exceeding 150, projected KrF-laser IFE efficiencies are consistent with a viable power plant.

For DPSSLs the beam smoothing is not as effective as for $\mathrm{KrF}$, and the fundamental wavelength is $1053 \mathrm{~nm}$. As result, the frequency must be tripled for ICF/IFE applications, and beam smoothing presents an important challenge. The near-term challenge facing the DPSSL approach involves discovering ways to meet the $7 \%$ efficiency requirement at the tripled frequency and to improve beam smoothing at progressively higher energy levels. This will be done along with specific research aimed at understanding damage resistance of key semiconductor optical components.

Turning to targets, the most important near-term issue is Rayleigh-Taylor instabilities seeded by non-uniformities in the laser deposition profile or in the target. This hydrodynamic instability must be suppressed sufficiently to allow the required high gains from the direct-drive target.

Recent innovations hold promise for mitigating the impact of hydrodynamic instabilities, including using high- $\mathrm{Z}$ target coatings to reduce beam imprint, and tailoring the target's postshock entropy profile. Simulations and experiments indicate that a carefully designed laser prepulse shapes the entropy profile and reduces the instability growth such that high gain is predicted for direct-drive targets.

In terms of immediate research plans, adequate resources and facilities are in place to address these near-term issues. The DPPSL and KrF laser programs have complete working facilities and supporting theoretical programs, and anticipate that these issues will be resolved on these systems 
within the next 2-3 years, given continued support of the NNSA/HAPL program. The target issues, including fabrication challenges, will be addressed as part of the ICF program using 2D and 3D codes and experiments on Nike, OMEGA and, when it becomes available, NIF.

Since the target issues are largely being addressed in the ICF program, the most critical near-term issue facing the HAPL program is:

Durability of KrF lasers, and efficiency and beam smoothing in DPSSLs, that will scale to the high-energy requirements for IFE.

\subsection{Z-Pinch Fusion}

For the Z-Pinch, the critical near-term issue involves the need for a direct physical connection between the pulsed-power supply and the Z-Pinch assembly "target" containing the fusion fuel capsule. This is in contrast to HI or HAPL where capsules are repetitively injected into the chamber and illuminated by multiple driver beams, but without any physical contact between driver and target. The Z-Pinch concept addresses the "direct connection" problem by means of a recyclable transmission line (RTL). On each shot the RTL will be destroyed, but the RTL materials will be collected and continually recycled. A new RTL will be inserted for each shot, with a repetition rate of about once every 10 seconds. Achieving this capability will require the resolution of both scientific and engineering issues.

The key scientific issues for Z-Pinch IFE are related to the power flow in the RTL. The Z machine uses high-power-density transmission lines that work well at the 20-MA level. However, IFE will require $\sim 60 \mathrm{MA}$ or more. Understanding the fundamental limits to power flow in the RTL is the main scientific concern as the currents are raised to IFE levels. Therefore, RTL research will be the focus in FY04 and for several years. The initial RTL experiments can be designed with existing codes (e.g. the ALEGRA MHD code, the LSP hybrid code, and the Quicksilver PIC code) and performed on the Saturn and Z facilities.

Thus, for the Z-Pinch approach to IFE the most important near-term issue is:

Physics limitations on power flow in a recyclable transmission line, including the coupling to the pulsed-power driver and the integral target assembly.

\subsection{Fast Ignition}

The most critical near-term goal for Fast Ignition is developing an approach to assembling a compressed sphere of fuel of uniform density and then efficiently heating a small part of that sphere under conditions that scale to ignition. The method of achieving the ideal state of a uniform-density sphere has not yet been found, even without the complexity of a cone imbedded in the target. A petawatt igniter laser beam will strongly interact with the plasma surrounding the compressed fuel, and convert about $40 \%$ of its energy to a relativistic electron beam. However, the HEDP issues of the beam transport to the fuel and energy deposition in the fuel are not well understood.

In the next several years, research can be carried out on existing or planned facilities to address critical feasibility issues for Fast Ignition. Numerical codes to design targets for fuel assembly 
exist. Codes to design and interpret energy transport experiments for the igniter-generated beam are required although some aspects of the physics can be addressed today. Plans for FI research include using existing facilities such as OMEGA, Z, and GEKKO in Japan, and then using FIREX1, OMEGA EP, and NIF in the future. Multi-kilojoule petawatt lasers coupled to implosion facilities should be available on OMEGA, Z, and GEKKO in the next 5 years.

Thus, the most critical near-term issue facing the Fast Ignition concept is:

Physics of fuel compression to a uniform-density sphere and of energy transport by relativistic electrons to that high-density fuel to achieve ignition. 
March 29, 2004

\section{POTENTIAL CONTRIBUTIONS TO HIGH-ENERGY-DENSITY PHYSICS AND OTHER SCIENCE}

The study of physical systems in which various combinations of high temperature, density, ionic charge state, magnetic field, etc., result in pressures exceeding a megabar (equivalently, energy densities exceeding about $1 \mathrm{eV} /$ angstrom $^{3}$ ) is now commonly called high-energy-density physics (HEDP). As illustrated in Figure 3, high energy density situations arise in astrophysics and planetary physics, as well as in inertial fusion science. The conditions of interest to IFE range from warm dense matter, to hot very dense plasmas, to extremely hot (even relativistic) plasmas. A great variety of HEDP topics is being addressed at universities within the US and abroad. The National Research Council reports, "Frontiers in High Energy Density Physics - The X-Games of Contemporary Science" and "Connecting Quarks with the Cosmos," noted that the intellectual challenges of HEDP stem from the interplay of non-linear, non-perturbative, and in some cases relativistic, many-body phenomena. The interested reader may refer to the first of these documents for a more thorough discussion of many issues discussed below as well as other, related topics. The focus here is on the synergies of IFE/ICF research and HEDP, plus some other applications of IFE science and technology.

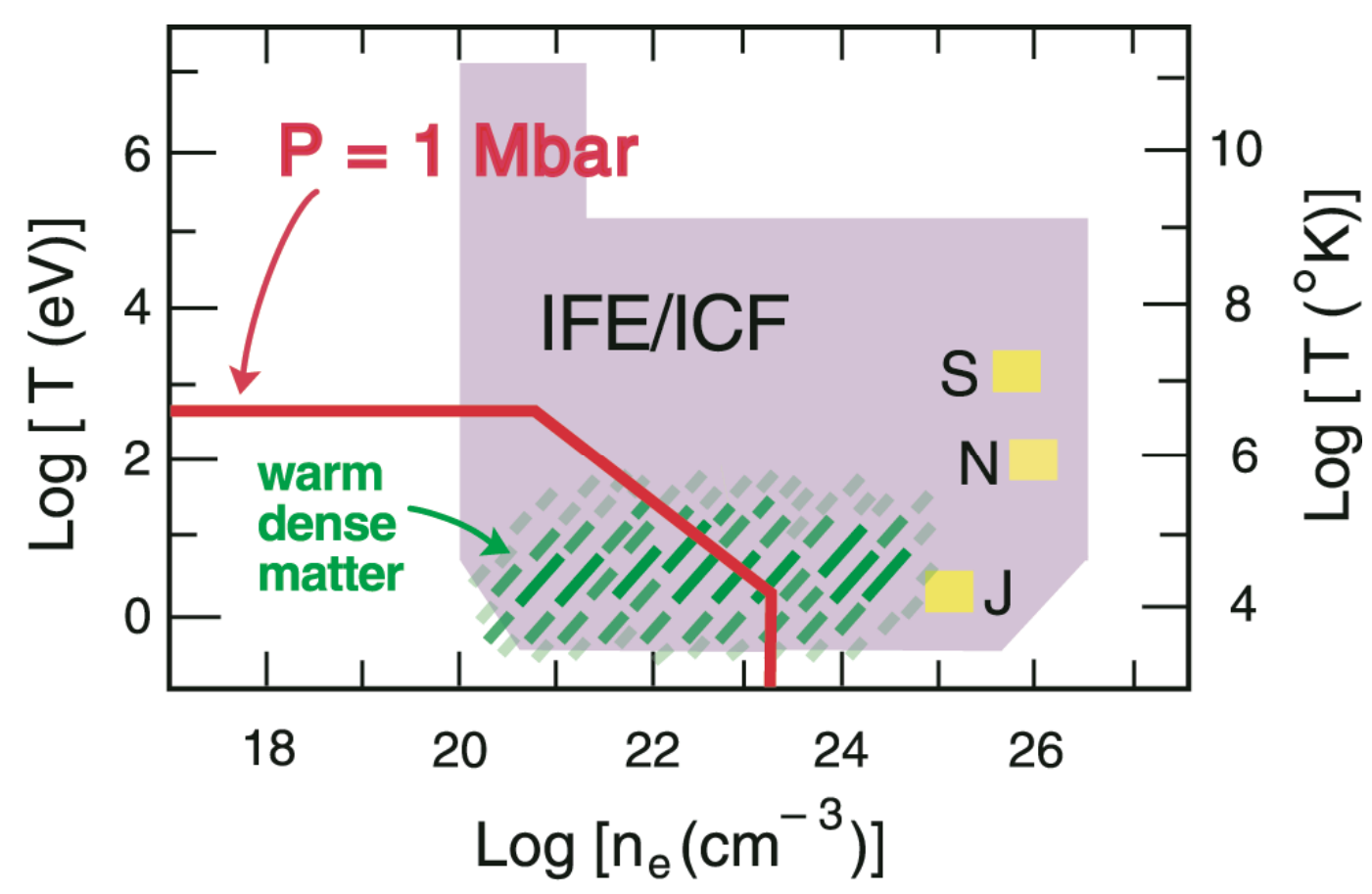

Figure 3. Some important high-energy-density regimes in the temperature-density plane. Above and to the right of the solid red line pressures exceed 1 Mbar; segments represent contributions due to radiation $\left(\mathrm{P} \sim \mathrm{T}^{4}\right)$, ideal gases $(\mathrm{P} \sim \mathrm{nT})$, and degenerate electrons $\left(\mathrm{P} \sim \mathrm{n}^{5 / 3}\right)$. The shaded region indicates the large range of conditions encountered or expected in some aspect of IFE/ICF research. Small squares identify astrophysical conditions characteristic of the cores of the sun (S) and Jupiter $(\mathrm{J})$, and the surface of a neutron star $(\mathrm{N})$. Warm dense matter lies within the hatched area. 
The ongoing development of the physics basis for fusion ignition and burn has been a driving force in a wide range of HEDP experiments. Substantial progress has been made within the NNSA-sponsored ICF program, but the need to achieve ignition, and then to achieve high gain in IFE targets, is pushing the limits of understanding of HEDP phenomena. An understanding of the physics of warm dense matter is also important for science and technology developments in the IFE and ICF programs and other aspects of the Stockpile Stewardship program, as well as planetary science and astrophysics. Thus there are natural synergies among topics in HEDP, in warm dense matter physics, and in the ICF and IFE programs.

The development of rapid-shot-rate capabilities is a requirement for IFE. As these capabilities become available they could be used to enhance the accessibility of experimental facilities used to study HEDP and warm dense matter regimes. Existing major facilities that are in high demand could be modified, or additional intermediate-sized facilities could be built that would enhance availability to a broader range of users. This "shot-on-demand" could potentially meet any rate that would be useful for research on such facilities, which would likely be less than several tens of shots per day compared with the many per minute required for IFE. This potential benefit to HEDP and other areas of science is described further in the sections that follow.

\subsection{IFE Synergies with High-Energy-Density Physics and Astrophysics}

There are numerous examples of HEDP phenomena being studied in the laboratory that also occur on cosmic scales [23]. Hydrodynamic instabilities similar to those observed in ICF afflict supernovae and have been modeled by similar numerical methods. The study of radiation transport in ICF-relevant regimes has resulted in improved understanding of radiative jets and related astrophysical phenomena. The need for better opacity data led to opacity experiments with iron that have improved models of Cepheid variables. The need for better equation-of-state (EOS) data for hydrogen is leading to a wide body of research important for understanding the interiors of large gaseous planets and objects outside of our solar system [24].

High-energy petawatt lasers can potentially facilitate laboratory astrophysics experiments that probe matter in the presence of intense, quasi-static magnetic fields (B) such as found in certain white dwarf stars (B 10 $0^{8}$ Gauss), and in the crusts of some neutron stars (B 109 Gauss). Spectroscopic and EOS data for this regime are needed to validate astrophysical models that will be used to interpret data from present and future space observatories.

There are other astrophysical applications. Fast Ignition calls for laser fluxes of order $10^{20}$ $\mathrm{W} / \mathrm{cm}^{2}$, hence energy densities exceeding $10^{4} \mathrm{eV} / \mathrm{Angstrom}^{3}$ and relativistic electron quiver velocities, to create a multi-MeV electron beam that ignites the fuel. Such laser fluxes would open up new regimes of laser-plasma interaction that should have astrophysical relevance. For instance, it has been suggested [25] that the Weibel instability of such beams is also responsible for the magnetic fields of relativistic collisionless shocks in gamma-ray bursters. This astrophysical instability has been studied with codes that were developed for laser-driven plasmas [26].

The dynamic response of warm dense matter to several Mbar pressures, temperatures of several hundred $\mathrm{eV}$, and with controlled megagauss-magnetic-field conditions can be investigated using the $\mathrm{Z}$ machine. As an example, recent studies have used lasers and the $\mathrm{Z}$ machine for accessing warm dense matter regions of the phase diagram of hydrogen and its isotopes that are inaccessible 
with other facilities [27,28]. This type of research could be conducted in a more timely way if the IFE-funded development of RTL technology for the Z machine and the HAPL technology are successful in providing shot-on-demand capabilities.

HI beams have a potential role in HEDP EOS studies since calculations suggest that properly tailored HI energy profiles produced in short pulses can heat solid targets with better than a few percent uniformity. If target thickness is short compared to the ion range, it is thought that short $\mathrm{HI}$ pulses could deposit energy in solid targets at energies near the Bragg peak, heating them at constant volume (isochoric heating). These experiments would thus be valuable for determining EOS properties of important materials along trajectories in pressure-temperature-density space corresponding to warm dense matter. This information is presently a critical need of the scientific community and would have a broad impact across several scientific disciplines and programmatic activities.

\subsection{IFE Synergies with the NNSA-funded ICF Program}

DPSSL and $\mathrm{KrF}$ lasers could be used to perform dense plasma experiments in support of ICF applications. Data are needed from EOS experiments for optimizing shock timing in ICF implosions, from studies of laser-plasma interactions, and from studies of plasma opacities, all of which provide a foundation for validating advanced ASCI material models. The improved statistics that arise from a large number of experiments should prove valuable. Such "shot-ondemand" capabilities may also be extended to studies needing short-pulse, high-energy pulses using either specially configured DPSSL or KrF lasers. Examples of HEDP topics for these capabilities include EOS [29], intense ion-beam generation and use [16,17], filamentation and nonlinear Brillouin scattering [30,31] saturation mechanisms for parametric instabilities [32,33] and many-body collisional effects in dense plasmas [34].

Near-term improvements in Electra potentially allow substantial advances in the energy and pulse shaping capabilities of Nike, which could benefit the NNSA ICF program. Because of the shoton-demand capability, HAPL can also contribute to the development and testing of higher photon energy x-ray backlighting techniques that would be useful in ICF applications. An attractive feature of Mercury is that beam-plasma or ion-surface interaction experiments can be performed with infrared, green or blue, as well as ultraviolet light, which would be useful to fundamental studies of optical damage thresholds, testing of large area optical elements, and calibration of experimental diagnostics.

IFE target fabrication research can benefit the NNSA ICF program. Examples include: foam capsules envisioned for IFE high-yield targets that are now beginning to be used at OMEGA; IFE studies of DT material and thermal transport properties that are synergistic to an overall understanding of DT-ice behavior (and tritium handling in target systems) important for ICF targets; and IFE layering research with foam capsules that potentially has substantial impact on NIF indirect drive cryogenic capsule development. In the area of high-precision target placement, IFE injection techniques could potentially be utilized on NIF to reduce the amount of debris-producing material near the implosion. Progress toward the central objective of IFE target research - namely, producing low-cost targets with high-yield - may in the future lead to still more developments useful to the ICF program, as well as in HEDP research.

Development of armor for solid wall IFE chambers could provide a means of protecting high value components inside an ICF chamber of a high-yield facility such as NIF. Innovative energyabsorbing materials tested in the HAPL program already have been evaluated for use on NIF as 
beam dumps or localized debris traps. Methods of debris mitigation, such as magnetic deflection, could be applied to diagnostics or other sensitive in-chamber components.

From a complementary perspective, there also are a variety of ways that the ICF scientific program can continue to make major contribution to IFE efforts. The baseline approach to ignition on NIF is indirect drive. Since the capsule physics, as well as the physics of x-ray transport and energy balance within hohlraums, is largely independent of the source of x-rays, the process of reaching ignition on NIF would yield much of the critical target physics base for HI and Z-Pinch targets. NIF can also address the critical ignition physics for direct-drive targets essential for current laser IFE concepts when enhanced beam smoothing techniques are implemented. Valuable early results for direct-drive targets could be obtained with planar targets using a subset of NIF beams and possibly by carrying out polar direct-drive experiments on NIF. Following initial demonstration of ignition, there will be important experiments which can be done on NIF to test such issues as the performance of targets that could be mass manufactured for IFE applications. Several workshops [35-40] have identified a variety of IFE related experiments that could be carried out on NIF. In addition to potential experiments on NIF, other NNSA facilities also can be used to address important IFE target physics issues. For example, symmetry control techniques that are common between Z-Pinch targets and HI targets are now being studied on $\mathrm{Z}$.

The high-energy petawatt capabilities being implemented on Z, OMEGA, and NIF will be essential for developing the physics of Fast Ignition. If warranted NIF could be modified for a Fast Ignition demonstration.

\subsection{Potential IFE Contributions to Other Scientific Disciplines}

A wide variety of experimental studies of condensed matter under rapid loading is required for developing $a b$ initio theories of material response. A major issue in condensed-matter physics concerns development of complete equations of state to accurately describe material response. This requires accurate information in high-pressure, high-temperatures regions, as well as highpressure, low-temperature regions. Since many applications involve the transition of a solid from one crystalline form to another in nanosecond or shorter times, it is also important to understand the fundamental scientific issues involving the thermodynamic conditions and kinetics of how these polymorphic transitions occur.

The development of Recyclable Transmission Lines (RTL) on the Z machine would allow increased access that could potentially accelerate its use for condensed-matter physics studies, including the recently developed technique on $\mathrm{Z}$ for producing isentropic compression experiments that are unique in the high-pressure scientific community. This capability produces relatively cold states of matter to multi-Megabar pressures that far exceed the capabilities of any other existing technique, such as diamond anvil cells and gas guns. It has also proved useful in high-pressure isentropic EOS studies [41], studies of radiation defects on dynamic material response [42], and transformation kinetic studies of polymorphic phase transitions [41].

The development of more efficient pulsed-power sources through the IFE program (both Z-Pinch and HAPL) is also allowing development of compact generators for a variety of applications. Such systems have considerable commercial potential and a spin-off system from the $\mathrm{Z}$ machine for materials studies is already being marketed. 
The HI-beam accelerators being studied for IFE applications are proving useful for exploring gaselectron cloud effects caused by HI-beam losses in quadrupole transport channels in accelerators. These effects are important in accelerators used for a variety of nuclear physics studies, such as the structure of heavy nuclei and properties of rare isotopes produced when beam ions collide with ions in dense plasmas [43]. HI research also provides opportunities to investigate fundamental beam physics issues. These include determination of: (1) technical requirements for preserving high beam brightness during beam transport, (2) a basic understanding of beamplasma interactions for focusing beams, and (3) conditions for achieving longitudinal beam compression.

The very high beam uniformity of $\mathrm{KrF}$ lasers should be attractive for a broad range of scientific investigations including material science studies of large-scale thin-film optics coatings, and evaluation of optically induced laser damage mechanisms over large surfaces. Higher test rates and less expensive targets, both of which should evolve through the HAPL program, will allow fundamental discovery experiments for understanding basic physics and materials issues. Many aspects of the science and technology involved in the HAPL program are also applicable to the emerging field of extreme UV lithography for next-generation semiconductors. These aspects include: efficient high average power pulsed lasers, low-cost mass-produced targets, precise highthroughput target alignment, optimized laser-target coupling for efficient radiation production, and mitigation of debris from expanding laser plasmas.

Finding. IFE capabilities have the potential for significantly contributing to HEDP and other areas of science. Isochoric heating of substantial volumes to uniform, elevated temperatures should be achievable using HI beams. Investigations of the Fast Ignition concept can lead to exploration of exotic HEDP regimes. Moreover, the rapid turn-around capabilities envisioned for IFE drivers could accelerate progress in HEDP science by enabling a wide community of users to conduct "shot-on-demand" experiments with data rates and volumes far exceeding those obtained on large systems that currently require long times between shots.

Finding: The NNSA-funded ICF program has the vital role of achieving ignition, and its research on target physics offers tremendous leverage that allows the comparatively modest funding for IFE-specific programs to continue to yield important advances. Carrying out a coordinated IFE research program now will guide future experiments on OMEGA, Nike, Z, and soon NIF, to develop the advanced target designs that not only meet the IFE physics requirements of high gain, but also the IFE requirements for fabrication, injection, and recycling.

Finding: IFE research directly benefits the NNSA mission. A vigorous IFE research program will continue to foster innovation that may lead to improved NNSA capabilities in targets (design, fabrication, and characterization), driver performance, and chamber/optics components. 
March 29, 2004

\section{REFERENCES}

1. R. C. Davidson, chair, "Frontiers in High Energy Density Physics - The X-Games of Contemporary Science,” National Research Council Report (2003).

2. R.J. Goldston, et al., "A Plan for the Development of Fusion Energy," J. Fusion Energy 21, No. 2, pp 61-112.

3. N. A. Davies, Presentation given to FESAC, November 17, 2003.

4. C. Baker, et al., "Report of the FESAC Panel on Priorities and Balance" J. Fusion Energy 18, No.2, pp 65-84..

5. C. Baker, chair, "Report of the Panel on Criteria, Goals and Metrics," Final Report to FESAC, October 8, 1999.

6. R. Bangerter, G. Navratil, and N. Sautoff, co-chairs, "2002 Fusion Summer Study Report - Next Major Steps in Fusion,” J. Fusion Energy 20, No. 3, pp 75-88..

7. J.D. Sethian, et al., "Fusion energy with lasers, direct drive targets, and dry wall chambers," Nucl. Fusion 43 (2003) 1693-1709.

8. B.G. Logan, et al., "Heavy Ion Beam Science towards Inertial Fusion Energy," in Proceedings of the 19th International Conference on Plasma Physics and Controlled Fusion, IAEA, Lyon, France Oct 14-19, 2002.

9. J.D. Sethian, et al., "Electron Beam Pumped Krypton Fluoride Lasers for Fusion Energy," to be published in Proceedings of the IEEE, Special Issue on Pulsed Power: Technology and Applications, Edl Schamiloglu and Robert Barker guest editors, July, 2004.

10. D. Weidenheimer, et al., "Advanced Pulsed Power Concept and Component Development for KrF Laser IFE,” International Power Modulator Conference, Hollywood, CA, June 30-July 3, 2002.

11. R. H. Lehmberg and J. L. Giuliani, "Simulation of Amplified Spontaneous Emission in High Gain KrF Laser Amplifiers," Journal of Applied Physics, 94, 31 (2002).

12. G.E. Rochau, et al., "ZP-3, A Power Plant Utilizing Z-Pinch Fusion Technology," Inertial Fusion Sciences and Applications 2001, Elsevier (Editors: K. A. Tanaka, D. D. Meyerhofer, J. Meyer-ter-Vehn), 706 (2002).

13. S.A. Slutz, C.L. Olson, and Per Peterson, "Low mass recyclable transmission lines for Zpinch driven inertial fusion," Physics of Plasmas 10, 429 (2003).

14. R. Kodama, et al., "Fast heating of ultrahigh-density plasma as a step towards laser fusion ignition," Nature 412, 23 August 2001, pp 798-802. 
15. R. Kodama, et al., "Fast heating scalable to laser fusion ignition," Nature 418, 29 August 2002, pp 933-934.

16. R. A. Snavely, et al., "Proton Beam Focusing and Heating in Petawatt Laser-Solid Interactions," Proceedings of IFSA 2003.

17. P. Patel, et al., "Isochoric Heating of Solid Density Matter with an Ultrafast Proton Beam," Phys. Rev. Lett. 91, 125004 (2003).

18. S. S. Yu, et al., "An Updated Point Design for Heavy Ion Fusion,” Fusion Sci. Tech. 44 Sept. 2003, 266-273.

19. W. R. Meier et al., "Osiris and Sombrero Inertial Fusion Power Plant Designs," WJSA92-01, DOE/ER/54100-1, March 1992 (http://aries.ucsd.edu/LIB/REPORT/OTHER/OSIRISOMBRERO/).

20. F. Najmabadi, et al., "Assessment of Chamber Concepts for Inertial Fusion Energy Power Plants - The ARIES-IFE study," Inertial Fusion Science and Applications 2001, Kyoto Japan, Sept. 2001, 701-705.

21. C.D. Orth, S.A. Payne, and W.F. Krupke, "A diode pumped solid state laser driver for inertial fusion energy," Nuclear Fusion 36, Number 1, January 1996.

22. S. E. Bodner, et al., "High-Gain Direct-Drive Target Design for Laser Fusion," Physics of Plasmas 7(6), June 2000, pp. 2298-2301.

23. D.D. Ryutov and B.A. Remington, "Scaling astrophysical phenomena to high-energydensity laboratory experiments", Plasma Physics and Controlled Fusion 44, B407 (2002).

24. T. Guillot, "Interiors of Giant Planets Inside and Outside the Solar System." Science 286, 72 (1999).

25. M.V. Medvedev and A. Loeb, "Generation of magnetic fields in the relativistic shock of gamma ray burst sources”, Astrophysical J. 526, 697 (1999).

26. L.O. Silva, et al., "Interpenetrating Plasma Shells: Near-equipartition Magnetic Field Generation and Nonthermal Particle Acceleration." Astrophys. J. Lett. 596, L121 (2003).

27. G.W. Collins, et al., "Measurement of the equation of state of deuterium at the fluid insulator transition," Science 281, 1178 (1998).

28. M.D. Knudson, et al., "Equation of state of liquid deuterium to $70 \mathrm{GPa}$, Phys. Rev. Lett. 87, 225501-1 (2003).

29. Widmann, et al., "Interferometric investigation of femptosecond laser-heated expanded states," Physics of Plasmas 8, 3869 (2001).

30. D. Pesme, et al., "Resonant Instability of Laser Filaments in a Plasma," Phys Rev. Lett. 84, 278 (2000). 
31. D.E. Hinkel, et al., "National Ignition Facility targets driven at high radiation temperature: ignition, hydrodynamic instability, and laser-plasma interactions," Phys. Plasmas 11, 1128 (2002).

32. E.A. Williams, et al., "Effects of ion trapping on crossed-laser-beam stimulated Brillouin scattering," Phys. Plasmas 11, 231 (2004).

33. H.X. Vu, et al., "Kinetic inflation of stimulated Raman backscatter in regimes of high linear Landau damping," Phys. Plasmas 9, 1745 (2002).

34. M.S. Murillo and J.C. Weisheit, "Dense Plasmas, Screened Interactions, and Atomic Ionization,” Physics Reports 302, 1 (1998).

35. Tobin, et al., "The role of the National Ignition Facility (NIF) in developing Inertial Fusion Energy, Lawrence Livermore National Laboratory, UCRL0JC-114042-ABS, 1993.

36. B.G. Logan and M.T. Tobin "The Role of NIF in Developing Inertial Fusion Energy," Energy and Technology Review, Lawrence Livermore National Laboratory, Livermore, California, UCRL-52000-94-12, 33-42, (December 1994).

37. B.G. Logan, et al., "Utility of the U.S. National Ignition Facility for Development of Inertial Fusion Energy," Lawrence Livermore National Laboratory, Livermore, California, UCRL-JC-117018, IAEA-CN-60/B-P-15, (1994).

38. Tobin, et al., "Use of the National Ignition Facility for the Development of Inertial Fusion Energy," Lawrence Livermore National Laboratory, Livermore, California, UCRL-JC-117395, (1994).

39. Tobin, et al., "Contributions of the National Ignition Facility to the Development of Inertial Fusion Energy," Lawrence Livermore National Laboratory, Livermore, California, UCRL-JC-117698 Rev. 1, (1994).

40. M.T. Tobin, et al., "Utility of the National Ignition Facility for Inertial Fusion Energy and Radiation Sciences Experiments," Proc. 12th Topical Mtg. Technology of Fusion Energy (Reno, Nevada), June 16-20, 1996.

41. C.A. Hall, et al., "Experimental configuration for isentropic compression of solids using pulsed magnetic loading", Rev. Sci. Instr. 72, 3587 (2001).

42. D.B. Reisman, et al., "Isentropic compression of irradiated stainless steel on the $\mathrm{Z}$ Accelerator," J. Appl. Phys. 93, 8952 (2003).

43. OFES - German Government official agreement, "Implementing Agreement between the Department of Energy of the United States of America and the Federal Ministry of Education and Research of the Federal Republic of Germany“, The Department of Energy of the United States of America and the Federal Ministry of Education and Research of the Federal Republic of Germany. 
March 29, 2004

\section{Appendix A \\ Charge Letter}

July 17, 2003

Professor Richard Hazeltine, Chair

Fusion Energy Sciences Advisory Committee

Institute for Fusion Studies

University of Texas at Austin

Austin, TX 78712

Dear Professor Hazeltine:

In response to the considerable scientific and technical progress in the Inertial Fusion Energy (IFE) program during the past few years, I am requesting that FESAC carry out a review of DOE's IFE program to provide an assessment of the present status of the program. Due in part to Congressional action, IFE relevant programs reside in both the Office of Science and in the Office of Defense Programs(DP) within NNSA, therefore, this charge is somewhat broader than those normally submitted to FESAC. Both SC and DP support this review and concur that it be carried out by FESAC.

The inertial path to fusion energy has been pursued by the Office of Fusion Energy Sciences (OFES) over the past 12 years. OFES has mostly funded the heavy ion beam driver and associated technologies component. DP as part of its Inertial Confinement Fusion (ICF) program, has funded high energy density physics facilities (including the National Ignition Facility) and the "target physics" relevant to ICF. The success of DP's ICF ignition program has always been viewed as a necessary precursor to the demonstration of IFE. Over the past four years, because of the strong DP laser program and DP's ICF efforts, Congress has added significant resources to the DP budget to develop the high average power laser (HAPL) driver. The DP program for the HAPL driver and related technology will have a long-term impact on the future development of IFE, and needs to be evaluated in the overall context of the Office of Science's IFE mission and program.

The specific topics to be addressed in this review are:

1. The current status of the scientific basis and related technology of each of the approaches to IFE, including an assessment of the quality of work being carried out in the programs.

2. Critical scientific issues identified in each of the approaches to IFE that would contribute to understanding the long-range potential of IFE.

3. The impact that fast ignition as a concept improvement may have on IFE.

4. The potential contribution of the various IFE program elements to the emerging field of High Energy Density Physics. 
The IFE approaches to be considered in this review are those involving heavy ion beam drivers, laser drivers and the " $Z$ " approach. Because of the breadth of the requested review, please use additional expertise outside of FESAC membership as required.

The Department is cognizant of the intense effort that FESAC has put forth during the last year and appreciates the time and energy expended by the individual members of both FESAC and its subcommittees. I will look forward to the Committee's report.

I would like to receive a final report by early 2004 .

\author{
Sincerely, \\ /s/ \\ Raymond L. Orbach \\ Director
}


March 29, 2004

\section{Appendix B}

March 30, 2004

Dr. Ray Orbach

Director, Office of Science

U.S. Department of Energy

1000 Independence Avenue, S.W.

Washington, D.C. 20585

Dear Dr. Orbach:

The Fusion Energy Sciences Advisory Committee (FESAC) has reviewed the enclosed report, "A Review of the Inertial Fusion Energy Program," and submits it to you with our full endorsement.

The Report responds to your letter of July 17, 2003, "requesting that FESAC carry out a review of DOE's Inertial Fusion Energy (IFE) program to provide an assessment of the present status of the program." Thus FESAC appointed a 20-member panel, chaired by Dr. Rulon Linford with Vice Chairs Dr. Jill Dahlburg and Dr. Riccardo Betti, and including scientists actively engaged in IFE research as well as scientists specializing in magnetic fusion research and in such neighboring fields as space physics and astrophysics. We are grateful to Dr. Linford and the panel for their valuable service.

A principal conclusion of the report is that "the quality of [IFE] science and engineering research is excellent." In this regard it is noteworthy that the Panel reserved its assessment of technical quality to those members not participating in the program. On the other hand, the panel noted that "the planned termination of technology Programs...is not consistent with their importance... and the Panel is concerned about the impact of this action."

The Panel considered in detail the three main approaches being studied in the IFE program: high-average-power lasers, heavy-ion accelerators, and Z-pinches, comparing their relative status and identifying the key scientific and technical issues for each. It also examined the Fast Ignition concept - an innovative scheme that could be used with various drivers.

The Panel considered the contributions of IFE to the relatively new science of High Energy Density Physics (HEDP). Finding that the IFE program is likely to bring important benefits to HEDP research, the panel identified specific contributions that appear especially accessible. The Report points out that IFE seems poised to make similarly important contributions to astrophysics.

The Panel unanimously concludes that "the IFE program is technically excellent and...contributes in ways that are noteworthy to the ongoing missions of the DOE." FESAC agrees with this conclusion, and would add that methods, concepts and results 
from IFE science have enriched many areas of fusion research, including magnetic fusion energy science.

Yours truly,

Richard Hazeltine

Chair, Fusion Energy Sciences Advisory Committee

\section{Enclosure}

cc: N. A. Davies FESAC 